\title{
Processes Underlying Human Performance
}

\author{
Lisanne Bainbridge \\ University College London \\ Michael C. Dorneich \\ Honeywell Laboratories
}

Two decades ago, a chapter on aviation with this title might have focused on physical aspects of human performance, on representing the control processes involved in flying. There has been such a fundamental change in our knowledge and techniques that this chapter focuses almost exclusively on cognitive processes. The main aims are to show that relatively few general principles underlie the huge amount of information relevant to interface design, and that context is a key concept in understanding human behavior.

Classical interface human factors/ergonomics consists of a collection of useful but mainly disparate facts and a simple model of the cognitive processes underlying behaviorthat these processes consist of independent information-decision-action or if-then units. (The combined term human factors/ergonomics is used, shortened to HF/E, because these terms have different shades of meaning in different countries. Cognitive processing is the unobservable processing between arrival of stimuli at the senses and initiating an action.) Classic HF/E tools are powerful aids for interface design, but they make an inadequate basis for designing to support complex tasks. Pilots and air traffic controllers are highly trained and able people. Their behavior is organized and goal-directed, and they add knowledge to the 
information given on an interface in two main cognitive activities: understanding what is happening, and working out what to do about it.

As the simple models of cognitive processes used in classic HF/E do not contain reminders about all the cognitive aspects of complex tasks, they do not provide a sufficient basis for supporting HF/E for these tasks. The aim of this chapter is to present simple concepts that could account for behavior in complex dynamic tasks and provide the basis for designing to support people doing these tasks. As the range of topics and data that could be covered is huge, the strategy is to indicate key principles by giving typical examples, rather than attempting completeness. This chapter does not present a detailed model for the cognitive processes suggested, or survey HF/E techniques, and it does not discuss collective work. The chapter offers four main sections, on simple use of interfaces; understanding, planning, and multitasking; learning, workload, and errors; and joint cognitive systems. The conclusion outlines how the fundamental nature of human cognitive processes underlies the difficulties met by $\mathrm{HF} / \mathrm{E}$ practitioners.

\section{USING THE INTERFACE, CLASSIC HF/E}

This chapter distinguishes between cognitive functions or goals, what is to be done, and cognitive processes, how these are done. This section starts with simple cognitive functions and processes underlying the use of displays and controls, on the interface between a person and the device the person is using. More complex functions of understanding and planning are discussed in the next main section.

The view is taken here that simple operations are affected by the context in which they are done. Someone does not press a button in isolation. For example, a pilot keys in a 
radio frequency as part of contacting air traffic control, as part of navigation, which is multitasked with checking for aircraft safety, and so on. From this point of view, an account of cognitive processes should start with complex tasks. However that is just too difficult. Here, the section started with the simple tasks involved in using an interface, and point out how even simple processes are affected by a wider context. The next main section builds up from this to discuss more complex tasks.

Five main cognitive functions are involved in using an interface:

- Discriminating a stimulus from a background, or from other possible stimuli. The process usually used for this is decision making.

- Perceiving "wholes." The main process here is integrating together parts of the sensory input.

- Naming.

- Choosing an action. The cognitive process by which the functions of naming, and choosing an action, are done (in simple tasks) is recoding, that is, translating from one representation to another, such as (shape $\rightarrow$ name), or (display $\rightarrow$ related control).

- Comparison, which may be done by a range of processes from simple to complex.

Because discriminating and integrating stimuli are usually done as the basis for naming or for choosing an action, it is often assumed that the processes for carrying out these functions are independent, input driven, and done in sequence. However, the discussion shows that these processes are not necessarily distinct, or done in sequence, and that they all involve use of context and knowledge. 
This section does not discuss displays and controls separately, as both involve all the functions and processing types. Getting information may involve making a movement such as visual search or accessing a computer display format, whereas making a movement involves getting information about it. The four subsections are on detecting and discriminating; visual integration; naming and simple action choices; and action execution.

\section{Detecting and Discriminating}

It might be thought, because the sense organs are separate from the brain, that at least basic sensory effectiveness, the initial reception of signals by the sense organs, would be a simple starting point, before considering the complexities that the brain can introduce such as naming a stimulus or choosing an action. However, sensing processes turn out not to be simple: There can be a large contribution of prior knowledge and present context.

This part of the chapter is in four subsections, on detecting, discriminating one signal from others that are present, or not present (absolute judgment), and sensory decisions. It is artificial to distinguish between sensory detection and discrimination, although they are discussed separately here, because they both involve (unconscious) decision making about what a stimulus is. In many real tasks, other factors have more effect on performance than any basic limits to sensory abilities. Nevertheless, it is useful to understand these sensory and perceptual processes, because they raise points that are general to all cognitive processing.

Detecting. Detection is one of those words that may be used to refer to different things. In this section it is used to mean sensing the presence of a stimulus against a blank background. Detecting the presence of light is an example. A human eye has the ultimate 
sensitivity to detect one photon of electromagnetic energy in the visible wavelengths. However, we can only detect at this level of sensitivity if we have been in complete darkness for about half an hour (Fig. 6.1). The eyes adapt 50 they are sensitive to a range of light intensities around the average (Fig. 6.2); this adaptation takes time. Adaptation allows the eyes to deal efficiently with a wide range of stimulus conditions, but it means that sensing is relative rather than absolute.

The two curves on the dark adaptation graph (Fig. 6.1) indicate that the eyes have two different sensing systems, one primarily for use at high, and the other for use at low, light intensities. These two systems have different properties. At higher levels of illumination the sensing cells are sensitive to color. There is one small area of the retina (the sensory surface inside the eye) that is best able to discriminate between spatial positions, and best able to detect stationary objects. The rest of the sensory surface (the periphery) is better at detecting moving than stationary objects. At lower levels of illumination intensity, the eyes see mainly in black and white, and peripheral vision is more sensitive for detecting position.

Therefore it is not possible to make a simple statement that "the sensitivity of the eves is ...." The sensitivity of the eyes depends on the environment (e.g., the average level of illumination) and on the stimulus (e.g., its movement, relative position, or color). The sensitivity of sense organs adapts to the environment and the task, so sensitivity does not have an absolute value independent of these influences. This means it is difficult to make numerical predictions about sensory performance in particular circumstances, without testing directly. 
However, it is possible to draw practical implications from the general trends in sensitivity. For example, it is important to design to Support both visual sensing systems in tasks that may be done in both high and low levels of illumination, such as flying. It is also sensible to design so that the most easily detected stimuli (the most "salient") are used for the most important signals. Visual salience depends not only on intensity but also on the color, movement, and position of the stimulus. Very salient stimuli attract attention; they override the usual mechanism for directing attention (see next main section). This means that very salient signals can be either useful as warning signals, or a nuisance as irrelevant distractions that interrupt the main task thinking.

Discriminating Between Stimuli. In this section the word discrimination is used to mean distinguishing between two (or more) stimuli. As with detection, the limits to our ability to discriminate between stimulus intensities are relative rather than absolute. The just noticeable difference between two stimuli is a ratio of the stimulus intensities. (There is a sophisticated modem debate about this, but it is not important for most practical applications.) The ratio is called the Weber fraction. Again, the size of this ratio depends on the environmental and task context. For example, in visual intensity discriminations, the amount of contrast needed to distinguish between two stimuli depends on the size of the object (more contrast is needed to see smaller objects) and on the level of background illumination (more contrast is needed to see objects in lower levels of background illumination).

The Weber fraction describes the difference between stimuli that can just be discriminated. When stimuli differ by larger amounts, the time needed to make the 
discrimination is affected by the same factors: Finer discriminations take longer, and visual discriminations can be made more quickly in higher levels of background illumination.

Touch and feel (muscle and joint receptor) discriminations are made when using a control. For example, a person using a knob with tapered sides may make three times more positioning errors than when using a knob with parallel sides (Hunt \& Warrick, 1957). Neither of the sides of a tapered knob actually points in the direction of the knob, so touch information from the sides is ambiguous.

Resistance in a control affects how easy it is to discriminate by feel between positions of the control. Performance in a tracking task, using controls with various types of resistance, shows that inertia makes performance worse, whereas elastic resistance can give the best results. This is because inertia is the same whatever the size of movement made, so it does not help in discriminating between movements. Elastic resistance, in contrast, varies with the size of movement, so gives additional information about the movements being made (Howland \& Noble, 1955).

Absolute Judgment. The Weber fraction describes the limit to our abilities to discriminate between two stimuli when they are both present. When two stimuli are next to each other we can, at least visually, make very fine discriminations in the right circumstances. However, our ability to distinguish between stimuli when only one of them is present is much more limited. This process is called absolute judgment. The judgment limits to our sensory abilities are known in general, for many senses and dimensions (Miller, 1956). These limits can be affected by several aspects of the task situation, such as the range of possible stimuli that may occur (Helson, 19(4). 
When only one stimulus is present, distinguishing it from others must be done by comparing it with mental representations of the other possible stimuli. So absolute judgment must involve knowledge and/or working memory. This is an example of a sensory discrimination process that has some processing characteristics in common with that are usually considered much more complex cognitive functions. There is not always a dear distinction between simple and complex tasks in the aspects of processing involved.

Although our ability to make absolute judgments is limited, it can be useful. For example, we can discriminate among eight different positions within a linear interval. This means that visual clutter on scale-and-pointer displays can be reduced; it is only necessary to place a scale marker at every 5 units that need to be distinguished. But our ability is not good enough to distinguish between 10 scale units without the help of an explicit marker.

In other cases, the limitations need to be taken into account in design. For example, we can only distinguish among 11 different color hues by absolute judgment. As we are very good at distinguishing between colors when they are next to each other, it can be easy to forget that color discrimination is limited when one color is seen alone. For example, a color display might use green-blue to represent one meaning (e.g., main water supply) and purpleblue with another meaning (e.g., emergency water supply). It might be possible to discriminate between these colors, and so use them as a basis for identifying meaning, when the colors are seen together, but not when they are seen alone. (Some discussion of meaning is given later.) 
Again, discrimination is a process in which the task context, in this case whether or not the stimuli occur together for comparison, has a strong effect on the cognitive processes involved and on our ability to make the discriminations.

Sensory Decision Making. Detections and discriminations involve decisions about whether the evidence reaching the brain is sufficient to justify deciding that a stimulus (difference) is present. For example, detection on a raw radar screen involves deciding whether a particular radar trace is a "blip" representing an aircraft, or something else that reflects radar waves. A particular trace may only be more or less likely to indicate an aircraft, so a decision has to be made in conditions of uncertainty. This sort of decision can be modeled by signal detection or statistical decision theory. Different techniques are now used in psychology, but this approach is convenient here because it distinguishes between the quality of the evidence and the observer's prior biases about decision outcomes.

Suppose that radar decisions are based on intensity, and that the frequencies with which different intensities have appeared on the radar screen when there was no aircraft present have been as shown in Fig. 6.3a at the top, whereas the intensities that have appeared when an aircraft was present are shown in Fig. 6.3a at the bottom. There is a range of intensities that occurred only when an aircraft was not present, a range of intensities that occurred only when an aircraft was present, and an intermediate range of intensities that occurred both when an aircraft was present and when it was not (Fig. 6.3b). How can someone make a decision when one of the intermediate intensities occurs? The decision is made on the basis of signal likelihood. The height of the curve above a particular intensity indicates how likely that intensity was to occur when there was or was not an aircraft. At the midpoint between the two frequency distributions, both possibilities are equally likely. 
Intensities less than this midpoint are more likely not to come from an aircraft, and intensities greater than this midpoint are more likely to come from an aircraft.

Note that when a stimulus is in this intermediate range, it is not always possible to be right about a decision. A person can decide a trace is not an aircraft when it actually is (a "miss"), or can decide it is an aircraft when it is not (a "false alarm"). These ways of being wrong are not called errors, because it is not mathematically possible always to be right when making uncertain decisions. The number of wrong decisions and the time to make the decision increase when signals, are more similar (overlap more).

Note that when the radar operator is making the decision, there is only one stimulus actually present, with one intensity. The two frequency distributions, against which this intensity is compared to make the decision, must be supplied from the operator's previous experience of radar signals, stored in the operator's knowledge base. Decisions are made by comparing the input stimulus ("bottom-up") with stored knowledge about the possibilities (“top-down”).

In addition to the uncertainty due to similarity between possible interpretations of a stimulus, the second major factor in this type of decision making is the importance or costs of the alternative outcomes. In the example just given, the person's decision criterion, the intensity at which the person changes from deciding "yes" to deciding "no," was the point at which both possibilities are equally likely. But suppose it is very important not to miss a signal — for instance, when radar watch keeping in an early warning system. Then it might he sensible to use the decision criterion in Fig. 6.4. This would increase the number of hits. It would also increase the number of false alarms, but this might be considered a small price to 
pay compared with the price of missing a detection. Alternatively, imagine people doing a job in which when they detect a signal they have to do a lot of work, and they are feeling lazy and not committed to their lob. Then they might move their decision criterion in the other direction, to minimize the number of hits.

This shift in decision criterion is called bias. Decision bias can be affected by probabilities and costs. The person's knowledge of the situation provides the task and personal expectations/probabilities and costs that are used in setting the biases, so again topdown processing influences sensory decisions. There are limits to human ability to assess biases (Kahneman, Slovic, \& Tversky, 1992). At extreme probabilities we tend to substitute determinacy for probability. We may think something is sure to happen, when it is just highly likely. Some accidents happen because people see what they expect to see, rather than what is actually there (e.g., Davis, 1966). Inversely, we may think something will never happen, when it is objectively of very low probability. For example, when signals are very unlikely, then it is difficult for a human being to continue to direct attention to watching for them (the "vigilance" effect).

\section{Visual Integration}

The effects of knowledge and context are even more evident in multidimensional aspects of visual perception, such as color, shape, size, and movement, in which what is seen is an inference from combined evidence. This discussion is in subsections on movement, size, and color; grouping processes; and shape. (There are also interesting auditory integrations, much involved in music perception, hut these are not discussed here.) 
Movement, Size, and Color Constancies. It is actually quite odd that we perceive a stable external world, given that we and other objects move, and the wavelength of the environmental light we see by changes, so the size, position, shape, and wavelength of light reflected from objects onto the retina all change. As we do perceive a stable world, this suggests our perception is relative rather than absolute: We do not see what is projected on the retina, but a construction based on this projection, made by combining evidence from different aspects of our sensory experience. The processes by which a wide variety of stimuli falling on the retina are perceived as the same are called constancies.

When we turn our heads the stimulation on the retina also moves. However, we do not see the world as moving, because information from the turning receptors in the ear is used to counteract the evidence of movement from the retina. The changes on the retina are perceived in the context of changes in the head rotation receptors. When the turning receptors are diseased, or the turning movements are too extreme for the receptors to be able to interpret quickly, then the person may perceive movement that is not actually occurring, as in some flying illusions.

There is also constancy in size perception. As someone walks away from us, we do not sec them becoming smaller and smaller, although there are large changes in the size of the image of that person that falls on the retina. In interpreting the size of objects, we take into account all the objects that are at the same distance from the eye, and then perceive them according to their relative size. Size constancy is more difficult to account for than movement constancy, as it involves distance perception, itself a complex process (Gibson, 1950). Distance is perceived by combining evidence about texture, perspective, changes in color of light with distance, and overlapping (itself a construct, discussed later). Information from the 
whole visual field is used in developing a percept that makes best overall sense of the combination of inputs. Cognitive psychology uses the concept that different aspects of stimulus processing are done simultaneously, unless an aspect is difficult and slows processing down. Each aspect of processing communicates its "results so far" to the other aspects via a "blackboard," and all aspects work together to produce a conclusion (Rumelhart, 1977).

Color perception is also an integrative process that shows constancy. Research on the color-receptive cells in the retina suggests that there are only three types of cell, which respond to red, green, and blue light wavelengths. The other colors we "see" are constructed by the brain, based on combinations of stimulus intensities at these three receptors. The eyes are more sensitive to some colors, so if a person looks at two lights of the same physical intensity but different wavelengths, the lights may be of different experienced intensity (brightness). The effectiveness of the color construction process is such that there are some visual demonstrations in which people see a range of colors, even though the display consists only of black and white plus one color. This constructive process also deals with color constancy. The wavelength of ambient lighting can change quite considerably, so the light reflected from objects also changes in wavelength, but objects are perceived as having stable color. The wavelengths of light from all the objects change in the same way, and color is perceived from the relative combinations of wavelengths, not the actual wavelengths. This constancy process is useful for perceiving a stable world despite transient and irrelevant changes in stimuli, but it does make designing color displays more difficult. As with our response to stimulus intensity, our perception of color is not a fixed quantity that can easily be defined and predicted. Instead, it depends on the interaction of several factors in the 
environment and task contexts, so it may be necessary to make color perception tests for a particular situation.

Grouping Processes. Another type of perceptual integration occurs when several constituents of a display are grouped together and perceived as a "whole." The Gestalt psychologists in the 1920s first described these grouping processes, which can be at several levels of complexity.

1. Separate elements can be seen as linked into a line or lines. There are four ways in which this can happen: when the elements are close together, are similar, lie on a line, or define a contour. The grouping processes of proximity and similarity can be used in the layout of displays and controls on a conventional interface, to show which items go together.

2. When separate elements move together they are seen as making a whole. This grouping process is more effective if the elements are also similar. This is used in the design of head-up displays and predictor displays, as in Fig. 6.5.

3. Something that has uniform color or a connected contour is seen as a "whole"-for example, the four sides of a square are seen as a single square, not as four separate element.

4. The strongest grouping process occurs when the connected contour has a "good" form, that is, a simple shape. For example, a pull-down menu on a computer screen is seen as a distinct unit in front of other material, because it is a simple shape, and the elements within the shape are similar and (usually) different from the elements on the rest of the screen. When the visual projections of two objects are touching, then the one with the simplest shape is usually seen as in front of (overlapping) the other. 
The visual processes by which shapes and unities are formed suggest recommendations for the design of symbols and icons that are easy to sec (Easterby, 1970).

Shape Constancy. Visual integrative processes ensure that we see a unity when there is an area of the same color, or a continuous contour. The shape we see depends on the angles of the contour lines (there are retinal cells which sense angle of line). Again there are constancy processes. The shape perceived is a construction, taking into account various aspects of the context, rather than a simple mapping of what is projected from the object onto the retina. Figure 6.6 shows a perspective drawing of a cube, with the same ellipse placed on each side. The ellipse on the front appears as an ellipse on a vertical surface. The ellipse on the top appears to be wider and sloping at the same angle as the top. The ellipse on the side is ambiguous - is it rotated, or not part of the cube at all? The ellipse on the top illustrates shape "constancy." It is perceived according to knowledge about how shapes look narrower when they are parallel to the line of sight, so a flat narrow shape is inferred to be wider. Again, the constancy process shows that the surrounding context (in this case the upper quadrilateral) affects how particular stimuli are seen.

The Gestalt psychologists provided dramatic examples of the effects of these inference processes, in their reversible figures as in Fig. 6.7. The overall interpretation that is given to this drawing affects how particular elements of it are grouped together and namedfor example, whether they are seen as parts of the body or pieces of clothing. It is not possible to see both interpretations at the same time, but it is possible to change quickly from one to the other. As the interpretation given to an object affects how parts of it are perceived, this can cause difficulty with the interpretation of low-quality visual displays, for example, from infrared cameras or on-board radar. 


\section{Naming, and Simple Action Choices}

The next functions to consider are identifying name, status, or size, and choosing the nature and size of actions. These cognitive functions may be met by a process of recoding (association) from one form of representation to another, such as:

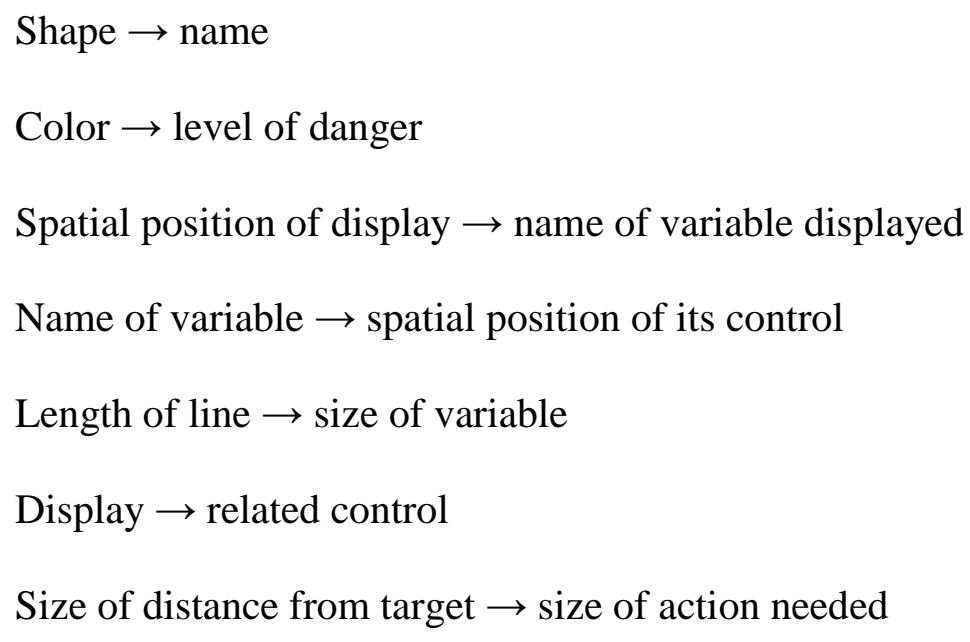

Identifications and action choices that involve more complex processing than this recoding are discussed in the section on complex tasks. This section discusses interdependence of the processes and functions; identifying name and status—-shape, color, and location (odes; size $\rightarrow$ size codes; and recoding/reaction times. Computer displays have led to the increased use of alphanumeric codes, which are not discussed here (see Bailey, 1989).

\section{Interdependence of the Functions}

Perceiving a stimulus, naming it, and choosing an action are not necessarily independent. Figure 6.7 shows that identification can affect perception. This section gives three examples that illustrate other HF/E issues. 
Naming difficulties can be based on discrimination difficulties. Figure 6.8 shows the signal/noise ratio needed to hear a word against background noise. The person listening not only has to detect a word against the noise background, but also has to discriminate it from other possible words. The more alternatives there are to distinguish, the better the signal/noise ratio needs to be. This is the reason for using a minimum number of standard messages in speech communication systems, and for designing these messages to maximize the differences between them, as in the International Phonetic alphabet, and standard airtraffic control language (Bailey, 1989).

An important aspect of maximizing differences between signals can be illustrated by a visual example. Figure 6.9 shows some data on reading errors with different digit designs. Errors can be up to twice as high with design A than with design $\mathrm{C}$ At a quick glance, these digit designs do not look very different, but each digit $\mathrm{In} \mathrm{C}$ has been designed to maximize its difference from the others. Digit reading is a naming task based on a discrimination task, and the discriminations are based on differences between the straight and curved elements of the digits. It is not possible to design an 8 that can be read easily, without considering the need to discriminate it from 3, 5, 6, and 9, which have elements in common. As a general principle, design for discrimination depends on knowing the ensemble of alternatives to be discriminated, and maximizing the differences between them.

However ease of detection/discrimination does not necessarily make naming easy. Figure 6.10 shows an iconic display. Each axis displays a different variable, and when all eight variables are on target, the shape is symmetrical. It is easy to detect a distortion in the shape, to detect that a variable is off target. However, studies show that people have difficulty with discriminating one distorted pattern from another by memory, and with identifying 
which pattern is associated with which problem. This display supports detection, but not discrimination or naming. It is important in task analysis to note which of the cognitive functions are needed, and that the display design supports them.

\section{Shape, Color, and Location Codes for Name and Status}

Conventional interfaces all too often consist of a sea of displays or controls that are identical both to sight and touch. The only way of discriminating between and identifying them is to read the label or learn the position Even if labels have well-designed typeface, abbreviations, and position, they are not ideal. What is needed is an easily seen "code" for the name or status, which is easy to recode into its meaning. The codes used most frequently are shape, color, and location, (Felt texture can be an important code in the design of controls.) The codes need to be designed for ease of discrimination, and for ease of making the translation from code to meaning.

Shape Codes. Good shape codes are "good" figures in the Gestalt sense, and also have features that make the alternatives easy to discriminate. However, ease of discrimination is not the primary criterion in good shape code design. Figure 6.11 shows the materials used in discrimination tests between sets of colors, military look-alike shapes, geometric forms, and aircraft look-alike shapes. Color discrimination is easiest, military symbols are easier to distinguish than aircraft symbols because they have more different features, and geometric forms are discriminated more easily than aircraft shapes. (Geometric forms are not necessarily easier to discriminate. For example, the results would be different if the shapes included an octagon as well as a circle.) The results from naming tests rather than discrimination tests would be different if geometric shapes or colors had to be given a 
military or aircraft name. Naming tests favor look-alike shapes, as look-alike shapes can be more obvious in meaning.

Nevertheless, using a look-alike shape (symbol or icon) does not guarantee obviousness of meaning. That people make the correct link from shape to meaning needs to be tested carefully. People can be asked, for each possible shape, what they think it is a picture of; what further meaning, such as an action, they think it represents; and, given a list of possible meanings, which of these meanings they choose as the meaning of the shape. To minimize confusions when using shape codes, it is important not to include in the coding vocabulary any shape that is assigned several meanings, or several shapes that could all be assigned the same meaning. Otherwise there could be high error rates in learning and using the shape codes. It is also important to test these meanings on the appropriate users, naive or expert people, or an international population. For example, in Britain a favored symbol for "delete" would be a picture of a space villain from a children's TV series, but this is not understood by people from other European countries!

As well as the potential obviousness of their meaning, look-alike shapes have other advantages over geometric shapes. They can act as a cue to a whole range of remembered knowledge about this type of object (see later discussion on knowledge). Look-alike shapes can also vary widely, whereas the number of alternative geometric shapes which are easy to discriminate is small. An interface designer using geometric shape as a code runs out of different shapes quite quickly, and may have to use the same shape with several meanings. The result of this is that a person interpreting these shapes has to notice when the context has changed to one in which a different shape $\rightarrow$ meaning translation is used, and then to remember this different translation, before the person can work out what a given shape means. 
This multistage process can be error prone, particularly under stress. Some computer-based displays have the same shape used with different meanings in different areas of the same display. A person using such a display has to remember to change the coding translation used every time the person makes an eye movement.

Color Codes. Using color as a code poses the same problems as using geometric shape. Except for certain culture-based meanings, such as red $\rightarrow$ danger, the meanings of colors have to be learned specifically, rather than being obvious. And only a limited number of colors can be discriminated by absolute judgment. The result is that a designer who thinks color is easy to see rapidly runs out of different colors, and has to use the same color with several meanings. There are computer-based displays on which color is used simultaneously with many different types of meaning, such as:

$$
\begin{aligned}
& \text { Color } \rightarrow \text { substance (steam, oil, etc.) } \\
& \text { Color } \rightarrow \text { status of item } \mathrm{kg} ., \text { on/off) } \\
& \text { Color } \rightarrow \text { function of item } \\
& \text { Color } \rightarrow \text { subsystem item belongs to } \\
& \text { Color } \rightarrow \text { level of danger } \\
& \text { Color } \rightarrow \text { attend to this item } \\
& \text { Color } \rightarrow \text { click here for more information } \\
& \text { Color } \rightarrow \text { click here to make an action }
\end{aligned}
$$

A user has to remember which of these coding translations is relevant to a particular point on the screen, with a high possibility of confusion errors 
Location Codes. The location of an item can be used as a basis both for identifying an item and for indicating its links with other items.

People can learn where a given item is located on an interface, and then look or reach to it automatically, without searching. This increases the efficiency of behavior. But this learning is effective only if the location $\rightarrow$ identity mapping remains constant; otherwise there can be a high error rate. For example, Fitts and Jones (1947/1961a), in their study of pilot errors, found that $50 \%$ of errors in operating aircraft controls were choosing the wrong control. The layout of controls on three of the aircraft used at that time shows why it was easy to be confused:

\begin{tabular}{|c|c|c|c|}
\hline \multirow[b]{2}{*}{ Aircraft } & \multicolumn{3}{|c|}{ Position of Control } \\
\hline & Left & Center & Right \\
\hline B-25 & Throttle & Prop & Mixture \\
\hline C-47 & Prop & Throttle & Mixture \\
\hline $\mathrm{C}-82$ & Mixture & Throttle & Prop \\
\hline
\end{tabular}

Suppose $\mathrm{n}$ pilot had flown a B-25 sufficiently frequently to be able to reach to the correct control without thinking or looking. If he then transferred to a C-17, two thirds of his automatic reaches would be wrong, and if to a C-82, all of them. As with other types of coding, location $\rightarrow$ identity translations need to be consistent and unambiguous Locations will be easier to learn if related items are grouped together, such as items from the same part of the device, with the same function, or the same urgency of meaning.

Locations can sometimes have a realistic meaning, rather than an arbitrary learned one. Items on one side in the real world should be on the same side when represented on an 
interface. (Ambiguity about the location of left/right displays could have contributed to the Kegworth air crash; Green, 1990.) Another approach is to put items in meaningful relative positions. For example, in a mimic/schematic diagram or an electrical wiring diagram, the links between items represent actual flows from one part of the device to another. On a cause-effect diagram, links between the nodes of the diagram represent causal links in the device. On such diagrams relative position is meaningful, and inferences can be drawn from the links portrayed (see later discussion on knowledge).

Relative location can also be used to indicate which control goes with which display. When there is a one-to-one relation between displays and controls, then choice of control is a recoding that can be made more or less obvious, consistent, and unambiguous by the use of spatial layout. Gestalt proximity processes link items together if they are next to each other. But the link to make can be ambiguous, such as in the layout: O O O O X X X X. Which X goes with which $\mathrm{O}$ ? People bring expectations about code meanings to their use of an interface. If these expectations are consistent among a particular group of people, the expectations are called population stereotypes. If an interface uses codings that are not compatible with a person's expectations, then the person is likely to make errors.

If two layouts to be linked together are not the same, then studies show that reversed but regular links are easier to deal with than random links (Fig. 6.12). This suggests recoding may be done, not by learning individual pairings, but by having a general rule from which one can work out the linkage.

In multiplexed computer-based display systems, in which several alternative display formats may appear on the same screen, there are at least two problems with location coding. 
One is that each format may have a different layout of items. We do not know whether people can learn locations on more than one screen format sufficiently well to be able to find items on each format by automatic eve movements rather than by visual search. If people have to search a format for the item they need, studies suggest this could take at least $25 \mathrm{sec}$. This means that every time the display format is changed, performance will be slowed down while this search process interrupts the thinking about the main task (see also later discussion on short-tern. memory). It may not be possible to put items in the same absolute position on each display format, but one way of reducing the problems caused by inconsistent locations is to locate items in the same relative positions on different formats.

The second location problem in multiplexed display systems is that people need to know the search "space" of alternative formats available, where they currently are in it, and how to get to other formats. It takes ingenuity to design so that the user of a computer-based interface can use the same sort of "automatic" search skills for obtaining information that are possible with a conventional interface.

In fact, there can be problems with maximizing the consistency and reducing the ambiguity of all types of coding used on multiple display formats (Bainbridge, 1991). Several of the coding vocabularies and coding translations used may change between and within each format (beware the codes used in figures in this chapter). The cues a person uses to recognize which coding translations are relevant need to be learned, and are also often not consistent. A display format may have been designed so the codes are obvious in meaning for a particular subtask, when the display format and the subtask are tested in isolation. But when this display is used in the real task, before and after other formats used for other subtasks, each of which 
uses different coding translations, then a task-specific display may not reduce either the cognitive processing required or the error rates,

\section{Size $\rightarrow$ Size Codes}

On an analogue interface, length of line is usually used to represent the size of a variable. The following arguments apply both to display scales and to the way control settings are shown. There are three aspects: the ratio of the size on the interface to the size of the actual variable; the way comparisons between sizes are made; and the meaning of the direction of a change in size.

Interface Size: Actual Size Ratio. An example of the interface size to actual size ratio is that, when using an analogue control (such as a throttle), a given size of action has a given size of effect. Once people have learned this ratio, they can make actions without having to check their effect, which gives increased efficiency (see later discussion).

The size ratio and direction of movement are again codes used with meanings that need to be consistent. Size ratios can cause display reading confusions if many displays are used, which all look the same but differ in the scaling ratio used. If many controls that are similar in appearance and feel are used with different control ratios, then it may be difficult to learn automatic skills in using them to make actions of the correct size. This confusion could be increased by using one multipurpose control, such as a mouse or tracker ball, for several different actions each with a different ratio.

A comparison of alternative altimeter designs is an example that also raises some general HF/E points. The designs were tested for reading speed and accuracy (Fig. 6.13). The 
digital display gives the best performance, and the three-pointer design (A) is one of the worst. The three-pointer altimeter poses several coding problems for someone reading it. The three pointers are not clearly discriminable. Each pointer is read against the same scale using a different scale ratio, and the size of pointer and size of scale ratio are inversely related (the smallest pointer indicates the largest scale, 10,000 s, the largest pointer $100 \mathrm{~s}$ ).

Despite these results, a digital display is not now used. A static reading test is not a good reflection of the real flying task. In the real task, altitude changes rapidly so a digital display would be unreadable. And the user also needs to identify rate of change, for which angle of line is an effective display. Unambiguous combination altimeter displays are now used, with a pointer for rapidly changing small numbers, and a digital display for slowly changing large numbers (D). Before this change, many hundreds of deaths were attributed to misreadings of the three-pointer altimeter, yet the display design was not changed until these comparative tests were repeated two decades later. This delay occurred for two reasons, which illustrate that $\mathrm{HF} / \mathrm{E}$ decisions are made in several wider contexts. First was the technology: In the 1940s, digital instrument design was very much more unreliable than the unreliability of the pilot's instrument readings. Second, cultural factors influence the attribution of responsibility for error. There is a recurring swing in attitudes, between saying that a user can read the instrument correctly so the user is responsible for incorrect readings, to saying that if a designer gives users an instrument that it is humanly impossible to read reliably, then the responsibility for misreading errors lies with the designer.

Making Comparisons Between Sizes. There are two important comparisons in control tasks: Is the variable value acceptable/within tolerance (a check reading), and if not, how big is the error? These comparisons can both usually be done more easily on an analogue display. 
Check readings can be made automatically (i.e., without processing that uses cognitive capacity) if the pointer on a scale is in an easily recognizable position when the value is correct. And linking the size of error to the sire of action needed to correct it can be done easily if both are coded by length of line.

An example shows why it is useful to distinguish cognitive functions from the cognitive processes used to meet them. Comparison is a cognitive function that may be done either by simple recoding or by a great deal of cognitive processing, depending on the display design. Consider the horizontal bars in Fig. 6.13 as a display from which an HF/E designer must get information about the relative effectiveness of the altimeter designs. The cognitive processes needed involve searching for the shortest performance bar by comparing each of the performance bar lines, probably using iconic (visual) memory, and storing the result in working memory, then repeating to find the next smallest, and so on. Visual and working memory is used as temporary working spaces while making the comparisons; working memory is also used to maintain the list of decision results. This figure is not the most effective way of conveying a message about alternative designs, because most people do not bother to do all this mental work. The same results are presented in Fig. 6.14. For a person who is familiar with graphs, the comparisons are inherent in this representation. A person looking at this does not have to do cognitive processing that uses processing capacity and is unrelated to and interrupts the main task of thinking about choice of displays. (See later discussion for more on memory interruption and on processing capacity.) This point applies in general to analogue and digital displays. For many comparison tasks, digital displays require more use of cognitive processing and working memory. 
Direction of Movement $\rightarrow$ Meaning. The second aspect to be learned about interface sizes is the meaning of the direction of a change in size. Cultural learning is involved here, and can be quite context specific. For example, people in technological cultures know that clockwise movement on a display indicates increase, but on a tap or valve control means closure, therefore decrease. Again there can be population stereotypes in the expectations people bring to a situation, and if linkages are not compatible with these assumptions, error rates may be at least doubled.

Directions of movements are often paired. For example, making a control action to correct a displayed error involves two directions of movement, on the display and on the control. It can be straightforward to make the two movements compatible in direction if both are linear, or both are circular.

It is in combining three or more movements that it is easy to get into difficulties with compatibility. One classic example is the aircraft attitude indicator. In the Fitts and Jones (1947/1961b) study of pilots' instrument reading errors, $22 \%$ of errors were either reversed spatial interpretations, or attitude illusions. In the design of the attitude indicator, four movements are involved: of the external world, of the display, of the control, and of the pilot's turning receptors (see Fig. 6.15). The attitude instrument can show a moving aircraft, in which case the display movement is the same as the joystick control movement but opposite to the movement of the external world. Or the instrument can show a moving horizon, which is compatible with the view of the external world but not with the movement of the joystick. There is no solution in which all three movements are the same, so some performance errors or delays are inevitable. Similar problems arise in the design of moving scales and of remote-control manipulation devices. 


\section{Reaction Times}

The evidence quoted so far about recoding has focused on error rates. The time taken to translate from one code representation to another also gives interesting information. Teichner and Krebs (1974) reviewed the results of reaction time studies. Figure 6.16 shows the effect of the number of alternative items and the nature of the recoding. The effect of spatial layout was illustrated in Fig. 6.12. Teichner and Krebs also reviewed evidence that, although unpracticed reaction times are affected by the number of alternatives to choose between, after large amounts of practice this effect disappears and all choices are made equally quickly. This suggests that response choice has become automatic; it no longer requires processing capacity.

The results show the effect of different code translations: using spatial locations of signals and responses (light, key) or symbolic ones (visually presented digit, spoken digit, i.e., voice). The time taken to make a digit $\rightarrow$ voice translation is constant, but this is already a highly practiced response for the people tested. Otherwise, making a spatial link (light $\rightarrow$ key) is quickest. Making a link that involves a change of code type, between spatial and symbolic (digit $\rightarrow$ key, or Light $\rightarrow$ voice), takes longer. (So these data show it can be quicker to locate than to name.) This coding time difference may arise because spatial and symbolic processes are handled by different areas of the brain, and it takes time to transmit information from one part of the brain to another. The brain does a large number of different types of coding translation (e.g., Barnard, 1987).

The findings presented so far come from studies of reacting to signals that are independent and occur one at a time. Giving advance information about the responses that 
will be required, which allows people to anticipate and prepare their responses, reduces response times. There are two ways of doing this, illustrated in Fig. 6.17. One is to give a preview, allowing people to see in advance the responses needed. This can more than halve reaction time. The second method is to have sequential relations in the material to be responded to. Figure 6.16 showed that reaction time is affected by the number of alternatives; the general effect underlying this is that reaction time depends on the probabilities of the alternatives. Sequential effects change the probabilities of items. One way of introducing sequential relations is to have meaningful sequences in the items, such as prose rather than random letters.

Reaction time and error rate are interrelated. Figure 6.18 shows that when someone reacts very quickly, the person chooses a response at random. As the person takes a longer time, and can take in more information before initiating a response, there is a trade-off between time and error rate. At longer reaction times there is a basic error rate that depends on the equipment used.

\section{Action Execution}

This chapter does not focus on physical activity, but this section makes some points about cognitive aspects of action execution. The section is in two parts, on acquisition movements, and on continuous control or tracking movements.

The speed, accuracy, and power a person can exert in a movement depend on its direction relative to the body position. Human biomechanics and its effects on physical performance, and the implications for workplace design, are large topics, which are not reviewed here (Pheasant, 1991). Only one point is made. Workplace design affects the 
amount of physical effort needed to make an action, and the amount of postural stress a person is under. These both affect whether a person is willing to make a particular action or to do a particular job. So workplace design can affect performance in cognitive tasks. Factors that affect what a person is or is not willing to do are discussed more in the section on workload.

Acquisition Movements. When someone reaches to something, or puts something in place, this is an acquisition movement. Reaching a particular endpoint or target is more important than the process of getting there. The relation between the speed and accuracy of these movements can be described by Fitts's Law (Fitts, 1954), in which movement time depends on the ratio of movement length to target width. However, detailed studies show that movements with the same ratio are not all carried out in the same way. Figure 6.19 shows that an $80 / 10$ movement is made with a single pulse of velocity. A 20/2.5 movement has a second velocity pulse, suggesting the person has sent a second instruction to his or her hand about how to move. Someone making a movement gives an initial instruction to his or her muscles about the direction, force, and duration needed, then monitors how the movement is being carried out, by vision and/or feel. If necessary the person sends a corrected instruction to the muscles to improve the performance, and so on. This monitoring and revision is called using feedback. A finer movement involves feedback to and a new instruction from the brain. A less accurate movement can be made with one instruction to the hand, without needing to revise it. An unrevised movement ("open-loop" or "ballistic") probably involves feedback within the muscles and spinal cord, but not visual feedback to and a new instruction from the brain. 
Movements that are consistently made the same way can be done without visual feedback, once learned, as mentioned in the section on location coding. Figure 6.20 shows the double use of feedback in this learning. A person chooses an action instruction that he or she expects will have the effect wanted. If the result turns out not to be as intended, then the person needs to adjust knowledge about the expected effect of an action. This revision continues each time the person makes an action, until the expected result is the same as the actual result. Then the person can make an action with minimal need to check that it is being carried out effectively. This reduces the amount of processing effort needed to make the movement. Knowledge about expected results is a type of meta-knowledge. Meta-knowledge is important in activity choice, and is discussed again later.

Control or Tracking Movements. Control movements are ones in which someone makes frequent adjustments, with the aim of keeping some part of the external world within required limits. They might be controlling the output of an industrial process, or keeping an aircraft straight and level. In industrial processes, the time lag between making an action and its full effect in the process may be anything from minutes to hours, so there is usually time to think about what to do. By contrast, in flying, events can happen very quickly, and human reaction lime plus neuromuscular lag, adding up to half a second or more, can have a considerable effect on performance. So different factors may be important in the two types of control task.

There are two ways of reducing the human response lag (cf. Fig. 6.17). Preview allows someone to prepare actions in advance and therefore to overcome the effect of the lag. People can also learn something about the behavior of the track they are following, and can then use this knowledge to anticipate what the track will do and so prepare their actions. 
There are two ways of displaying a tracking task. In a pursuit display, the moving target and the person's movements are displayed separately. A compensatory display system computes the difference between the target and the person's movements, and displays this difference relative to a fixed point. Many studies show human performance is better with a pursuit display, as in Fig. 6.21. As mentioned earlier, people can learn about the effects of their actions, and about target movements, and both types of learning lead to improved performance. On the pursuit display, the target and human movements are displayed separately, so a person using this display can do both types of learning. In contrast, the compensatory display only shows the difference between the two movements. It is not possible for the viewer to tell which part of a displayed change is due to target movements and which is due to the viewer's own movements, so these are difficult to learn.

A great deal is known about human fast tracking performance (Rouse, 1980; Sheridan \& Ferrell, 1974). A person doing a tracking task is acting as a controller. Control theory provides tools for describing some aspects of the track to be followed and how a device responds to inputs. This has resulted in the development of a "human transfer function," a description of a human controller as if the person were an engineered control device. The transfer function contains some components that describe human performance limits, and some that partially describe human ability to adapt to the properties of the device the person is controlling. This function can be used to predict combined pilot-aircraft performance. This is a powerful technique with considerable economic benefits. However, it is not relevant to this chapter as it describes performance, not the underlying processes, and it only describes human performance in compensatory tracking tasks. It also focuses attention on an aspect of human performance that can be poorer than that of fairly simple control devices. This 
encourages the idea of removing the person from the system, rather than appreciating what people can actively contribute, and designing support systems to overcome their limitations.

\section{Summary and Implications}

Theory. The cognitive processes underlying classic HF/E can be relatively simple, but not so simple that they can be ignored. Cognitive processing is carried out to meet cognitive functions. Five functions were discussed in this section: distinguishing between stimuli; building up a percept of an external world containing independent entities with stable properties; naming; choosing an action; and comparison.

This section suggests these functions could be met in simple tasks by three main cognitive processes. (What happens when these processes are not sufficient has been mentioned briefly and is discussed in the next main section.) The three processes are: deciding between alternative interpretations of the evidence; integrating data from all sensory sources, together with knowledge about the possibilities, into an inferred percept that makes best sense of all the information; and recoding, that is, translating from one type of code to another.

Five other key aspects of cognitive processing have been introduced:

1. Sensory processing is relative rather than absolute.

2. The cognitive functions are not necessarily met by processes in a clearly distinct sequence. Processes that are "automated" may be done in parallel. The processes communicate with each other via a common "blackboard," which provides the context within which each process works, as summarized in Fig. 6.22. 
As processing is affected by the context in which it is done, behavior is adaptive. However, for $\mathrm{HF} / \mathrm{E}$ practitioners this has the disadvantage that the answer to any HF/E question is always, "it depends."

3. The processing is not simply input driven: All types of processing involve the use of knowledge relevant in the context. (It can therefore be misleading to use the term knowledge-based to refer to one particular mode of processing.)

4. Preview and anticipation can improve performance.

5. Actions have associated meta-knowledge about their effects, which improves with learning.

Practical Aspects. The primary aim of classic HF/E has been to minimize unnecessary physical effort. The points made here emphasize the need to minimize unnecessary cognitive effort.

Task analysis should not only note which displays and controls are needed, but might also ask such questions as: What cognitive functions need to be carried out? By what processes? Is the information used in these processes salient?

In discrimination and integration: What is the ensemble of alternatives to be distinguished? Are the items designed to maximize the differences between them? What are the probabilities and costs of the alternatives? How does the user learn these?

In recoding: What coding vocabularies are used (shape, color, location, size, direction, alphanumeric) in each subtask? In the task as a whole? Are the translations unambiguous, 
unique, consistent, and if possible obvious? Do reaction times limit performance, and if so can preview or anticipation be provided?

\section{COMPLEX TASKS}

Using an interface for a simple task entails the functions of distinguishing between stimuli, integrating stimuli, naming, comparing, and choosing and making simple actions. When the interface is well designed, these functions can be carried out by decision making, integration, and recoding processes. These processes use knowledge about the alternatives that may occur, their distinguishing features, probabilities, and costs, and the translations to be made.

Doing a more complex task uses more complex knowledge in more complex functions and processes. For example, suppose an air traffic controller is given the two flight strips in Fig. 6.23. Commercial aircraft fly from one fix point to another. These two aircraft are flying at the same level $(31,000 \mathrm{ft})$ from fix OTK to fix LEESE7 DAL1152 is estimated to arrive at LEESE7 at 2 min after AALA19 $(18-16)$, and is traveling faster $(783>746)$. So DAL1152 is closing relatively fast and the controller needs to take immediate action, to tell one of the aircraft to change flight level. The person telling the aircraft to change level is doing more than simply recoding the given information. The person uses strategies for searching the displays and for comparing the data about the two aircraft, plus a simple dynamic model of how an aircraft changes position in time, to build up a mental picture of the relative positions of the aircraft, with one overtaking the other so a collision is possible. The person then uses a strategy for optimizing the choice of which aircraft to instruct to change. 
The overall cognitive functions or goals are to understand what is happening and to plan what to do about it. In complex dynamic tasks these two main cognitive needs are met by subsidiary cognitive functions such as:

- Infer/review present state.

- Predict/review future changes/events.

- Review/predict task performance criteria.

- Evaluate acceptability of present or future state.

- Define subtasks (task goals) to improve acceptability.

- Review available resources/actions, and their effects.

- Define possible (sequences of) actions (and enabling actions) and predict their effects.

- Choose action/plan.

- Formulate execution of action plan (including monitor the effects of actions, which may involve repeating all the preceding).

These cognitive functions are interdependent. They are not carried out in a fixed order but are used as necessary. Lower level cognitive functions implement higher level ones. At the lowest levels, the functions are fulfilled by cognitive processes such as searching for the information needed, discrimination, integration, and recoding. The processing is organized within the structure of cognitive goals/functions.

An overview is built up in working storage by carrying out these functions. This overview represents the person's understanding of the current state of the task and the person's thinking about it. The overview provides the data the person uses in later thinking, 
as well as the criteria for what best to do next and how best to do it. There is a cycle: Processing builds up the overview, which determines the next processing, which updates the overview, and so on (see Fig. 6.24). Figure 6.22 showed an alternative representation of context, as nested rather than cyclic. (For more about this mechanism, see Bainbridge 1993a.)

The main cognitive processes discussed in the previous section were decision making, integrating stimuli, and recoding. Additional modes of processing are needed in complex tasks, such as:

- Carrying out a sequence of recoding transformations, and temporarily storing intermediate results in working memory.

- Building up a structure of inference, an overview of the current state of understanding and plans, in working storage, using a familiar working method.

- Using working storage to menially simulate carrying out a cognitive or physical strategy.

- Deciding between alternative working methods on the basis of meta-knowledge.

- Planning and multitasking.

- Developing new working methods.

These complex cognitive processes are not directly observable. The classic experimental psychology method, which aims to control all except one or two measured variables, and to vary one or two variables so their effects can be studied, is well suited to investigating discrimination and recoding processes. It is not well suited to investigating cognitive activities in which many interrelated processes may occur without any observable behavior. Studying these tasks involves special techniques: case studies, videos, verbal protocols, or 
distorting the task in some way, perhaps slowing it down or making the person do extra actions to get information (Wilson \& Corlett, 1995). Both setting up and analyzing the results of such studies can take years of effort. The results tend to be as complex as the processes studied, so they are difficult to publish in the usual formats. Such studies do not fit well into the conventions about how research is done, so there are unfortunately not many of this type. However, the rest of this section gives some evidence about the nature of complex cognitive processes, to support the general claims made so far. The subsections are on sequences; language understanding; inference and diagnosis; working storage; planning, multitasking, and problem solving; and knowledge.

\section{Sequences of Transforms}

After decision making, integrating, and recoding, the next level of complexity in cognitive processing is carrying out $\mathrm{d}$ sequence of recoding translations or transforms. The result of one step in the sequence acts as the input to the next step, so has to be kept temporarily in working memory. Here the notion of recoding needs to be expanded to include transforms such as simple calculations and comparisons, and conditions leading to alternative sequences. Note that in this type of processing the goal of the behavior, the reason for doing it, is not included $\mathrm{m}$ the description of how it is done. Some people call this type of processing rulebased. There are two typical working situations in which behavior is not structured relative to goals.

When a person is following instructions that do not give them any reason for why the person has to do each action, then the person is using this type of processing. This is usually not a good way of presenting instructions, as if anything goes wrong, the person has no reference point for identifying how to correct the problem. 
The second case can arise in a stable environment, in which behavior can be done in the same way each time. If a person has practiced often, the behavior may be done without needing to check it, or to think out what to do or how to do it (see later discussion). Such overlearned sequences give a very efficient way of behaving, in the sense of using minimal cognitive effort. But if the environment does change, then overlearning is maladaptive and can lead to errors (see later discussion on learning and errors).

\section{Language Processing}

This section covers two issues: using language to convey information and instructions, and the processes involved in language understanding. Although language understanding is not the primary task of either pilot or air traffic controller, it does provide simple examples of some key concepts in complex cognitive processing.

Written Instructions. Providing written instructions is often thought of as a way of making a task easy, but this is not guaranteed. Reading instructions involves interpreting the words in order to build up a plan of action. The way the instructions are written may make this processing more or less difficult. Videorecorder operating manuals are notorious for this.

Various techniques have been used for measuring the difficulty of processing different sentence types. Some typical results are (Savin \& Perchonock, 1965):

\begin{tabular}{llc}
\hline Sentence Type & \multicolumn{1}{c}{ Example } & \% Drop in Performance \\
\hline Kernel & The pilot flew the plane. & 0 \\
Negative & The pilot did not fly the plane. & -16 \\
Passive & The plane was flown by the pilot. & -14
\end{tabular}


Such data suggest that understanding negatives and passives involves two extra and separate processes. This suggests it is best in general to use active positive forms of sentence. But when a negative or restriction is the important message, it should be the most salient and come first. For example, "No smoking" is more effective than "Smoking is not permitted," And using a simple form of sentence does not guarantee that a message makes good sense. I recently enjoyed staying in a hotel room with a notice on which the large letters said:

Do not use the elevator during a fire.

\section{Read this notice carefully.}

Connected prose is not necessarily the best format for showing alternatives in written instructions. Spatial layout can be used to show the groupings and relations between phrases, by putting each phrase on a separate line, by indenting to show items at the same level, and by using flow diagrams to show the effect of choice between alternatives (e.g., Oborne, 1995, chapter 4). When spatial layout is used to convey meaning in written instructions, it is a code and should be used consistently, as discussed earlier.

Instructions also need to be written from the point of view of the reader: "If you want to achieve this, then do this," Instruction books are often written the other way round: "If you do this, then this happens," The second approach requires from the reader much more understanding, searching, and planning to work out what to do. Note that the effective way of writing instructions is goal oriented. In complex tasks, methods of working are in general best organized in terms of what is to be achieved; this is discussed again later. 
Language Understanding. In complex tasks, many of the cognitive processes and knowledge used are only possible because the person has considerable experience of the task. Language understanding is the chief complex task studied by experimental psychologists (e.g., Ellis, 1993), because it is easy to find experts to test. When someone is listening to or reading language, each word evokes learned expectations. For example:

\author{
The \\ can only be followed by \\ - a descriptor, or \\ -a noun
}

\title{
The pilot
}

depending on the context, either;

(a) will be followed by the word "study" or:

(b) -evokes general knowledge (scenarios) about aircraft or ship pilots.

— can be followed by:

—a descriptive clause, containing items relevant to living things/animals/human beings/pilots, or

— a verb, describing possible actions by pilots.

Each word leads to expectations about what will come next; each constrains the syntax (grammar) and semantics (meaning) of the possible next words. To understand the language, a person needs to know the possible grammatical sequences, the semantic constraints on what words can be applied to what types of item, and the scenarios. During understanding, a 
person's working storage contains the general continuing scenario, the structure of understanding built up from the words received so far, and the momentary expectations about what will come next (Many jokes depend on not meeting these expectations.)

The overall context built up by a sequence of phrases can be used to disambiguate alternative meanings, such as:

\section{The Inquiry investigated why}

the pilot turned into a mountain.

or

\section{In this fantasy story \\ the pilot turned into a mountain.}

The knowledge base/scenario is also used to infer missing information. For example:

\section{The flight went to Moscow.}

\section{The stewardess brought her fur hat.}

Answering the question "Why did she bring her fur hat?" involves knowing that stewardesses go on flights and about the need for and materials used in protective clothing, which are not explicitly mentioned in the information given. 
Understanding language does not necessarily depend on the information being presented in a particular sequence. Although it requires more effort, we can understand someone whose first language uses a different word order from English, such as:

\section{The stewardess her fur hat brought.}

We do this by having a general concept that a sentence consists of several types of unit (noun phrases, verb phrases, and so on) and we make sense of the input by matching it to the possible types of unit. This type of processing can be represented as being organized by a "frame with slots," where the frame coordinates the slots for the types of item expected, which are then instantiated in a particular case, as in:

Noun phrase $\quad$ Verb Noun phrase

The stewardess brought her fur hat.

(As language has many alternative sequences, this is by no means a simple operation; Winograd, 1972.)

The understanding processes used in complex control and operation tasks show the same features that are found in language processing. The information obtained evokes both general scenarios and specific moment-to-moment expectations. The general context, and additional information, can be used to decide between alternative interpretations of the given information. A structure of understanding is built up in working storage. Frames or working methods suggest the types of information the person needs to look for to complete their understanding. These items can be obtained in a flexible sequence. And knowledge is used to 
infer whatever is needed to complete the understanding but is not supplied by the input information. There is an important addition in control/operation tasks, which is that the structure of understanding is built up in order to influence the state of the external world, to try to get it to behave in a particular way.

\section{Inference and Diagnosis}

To illustrate these cognitive processes in an aviation example, this section uses an imaginary example so the presentation can be short. Later sections describe real evidence on pilot and air traffic controller behavior, which justifies the claims made here.

Suppose that an aircraft is in flight and the "engine oil low" light goes on. What might be the pilot's thoughts? The pilot needs to infer the present state of the aircraft (cognitive functions are indicated by italics). This involves considering alternative hypotheses that could explain the light, such as that there is an instrument fault, or there genuinely is an engine fault, and then choosing between the hypotheses according to their probability (based on previous experience of this or other aircraft) or by looking for other evidence that would confirm or disprove the possibilities. The pilot could predict the future changes that will occur as a result of the chosen explanation of events. Experienced people's behavior in many dynamic tasks is future oriented. A person takes anticipatory action, not to correct the present situation, but to ensure that predicted unacceptable states or events do not occur. Before evaluating the predictions for their acceptability, the pilot needs to review the task performance criteria, such as the relative importance of arriving at the original destination quickly, safely, or cheaply. The result of comparing the predictions with the criteria will be to define the performance needs to be met. It is necessary to review the available resources, such as the state of the other engines or the availability of alternative landing strips. The pilot can then 
define possible alternative action sequences and predict their outcomes. A review of action choice criteria, which includes the task performance criteria plus others such as the difficulty of the proposed procedures, is needed as a basis for choosing an action sequence/plan, before beginning to implement the plan. Many of these cognitive functions must be based on incomplete evidence, for example, about future events or the effects of actions, so risky decision making is involved.

A pilot who has frequently practiced these cognitive functions may be able to carry them out "automatically," without being aware of the need for intermediate thought. And an experienced pilot may not be aware of thinking about the functions in separate stages; for example, (predict + review criteria + evaluation) may be done together.

Two modes of processing have been used in this example: "automatic" processing (i.e., recoding), and using a known working method that specifies what thinking to carry out. Other modes of processing are suggested later. The mode of processing needed to carry out a function depends on the task situation and the person's experience (see later discussion on learning). An experienced person's knowledge of the situation may (enable the person to reduce the amount of thinking to do, even when the person does need to think things out explicitly. For example, it may be clear early in the process of predicting the effects of possible actions that some will be not acceptable and so need not be explored further (see later discussion on planning).

Nearly all the functions and processing mentioned have been supplied from the pilot's knowledge base. The warning light evoked working methods for explaining the event and for choosing an action plan, as well as knowledge about the alternative explanations of events 
and suggestions of relevant information to look for. The combination of (working method + knowledge referred to in using this method + mental models for predicting events) is the scenario. Specific scenarios may be evoked by particular events, or by particular phases of the task (phases of the flight).

This account of the cognitive processes is goal oriented. The cognitive functions or goals are the means by which the task goals are met, but are not the same as them. Task and personal goals act as constraints on what it is appropriate and useful to think about when fulfilling the cognitive goals.

The cognitive functions and processing build up a structure of data (in working storage) that describes the present state and the reasons for it, predicted future changes, task performance and action choice criteria, resources available, the possible actions, the evaluations of the alternatives, and the chosen action plan. This data structure is an overview that represents the results of the thinking and deciding done so far, and provides the data and context for later thinking. As an example, the result of reviewing task performance criteria is not only an input to evaluation; it could also affect what is focused on in inferring the present state, or in reviewing resources, or in action choice. The overview ensures that behavior is adapted to its context.

The simple example just given described reaction to a single unexpected event. Normally, flying and air traffic control are ongoing task. For example, at the beginning of shift an air traffic controller has to build up an understanding of what is happening and what actions are necessary, from scratch. After this, each new aircraft that arrives is fitted into the controller's ongoing mental picture of what is happening in the airspace; the thinking 
processes do not start again from the beginning. Aircraft usually arrive according to schedule and are expected, but the overview needs to be updated and adapted to changing circumstances (see later discussion on planning and multitasking).

There are two groups of practical implications of these points. One is that cognitive task analysis should focus on the cognitive functions involved in a task, rather than simply prespecifying the cognitive processes by which they are met. The second is that designing specific displays for individual cognitive functions may be unhelpful. A person doing a complex task meets each function within an overall context, the functions are interdependent, and the person may not think about them in a prespecified sequence. Giving independent interface support to each cognitive function, or subtask within a function, could make it more difficult for the person to build up an overview that interrelates the different aspects of the person's thinking.

Diagnosis. The most difficult cases of inferring what underlies the given evidence may occur during fault diagnosis. A fault may be indicated by a warning light or, for an experienced person, by a device not behaving according to expectations. Like any other inference, fault diagnosis can be done by several modes of cognitive processing, depending on the circumstances. If a fault occurs frequently, and has unique symptoms, it may be possible to diagnose the fault by visual pattern recognition, that is, pattern on interface $\rightarrow$ fault identity (e.g., Marshall, Scanlon, Shepherd, \& Duncan, 1981). This is a type of recoding. But diagnosis can also pose the most difficult issues of inference, for example, by reasoning based on the physical or functional structure of the device (e.g., Hukki \& Norros, 1993). 
In-flight diagnosis may need to be done at speed. Experienced people can work rapidly using recognition-primed decisions, in which situations are assigned to a known category with a known response, on the basis of similarity. The processes involved in this are discussed by Klein (1989). The need for rapid processing emphasizes the importance of training for fault diagnosis.

Amalberti (1992, Expt. 4) studied fault diagnosis by pilots. Two groups of pilots were tested: Pilots in one group were experts on the Airbus, and those in the other group were experienced pilots beginning their training on the Airbus. They were asked to diagnose two faults specific to the Airbus, and two general problems. In $80 \%$ of responses, the pilots gave only one or two possible explanations. This is compatible with the need for rapid diagnosis. Diagnostic performance was better on the Airbus faults, which the pilots had been specifically trained to watch out for, than on the more general faults. One of the general problems was a windshear on take-off. More American than European pilots diagnosed this successfully. American pilots are more used to windshear as a problem, so are more likely to think of this as a probable explanation of an event. People's previous experience is the basis for the explanatory hypotheses they suggest.

In the second general fault there had been an engine fire on take-off, during which the crew forgot to retract the landing gear, which made the aircraft unstable when climbing. Most of the hypotheses suggested by the pilots to explain this instability were general problems with the aircraft, or were related to the climb phase. Amalberti suggested that when the aircraft changed the phase of flight, from take-off to climb, the pilots changed their scenario that provides the appropriate events, procedures, mental models, and performance criteria for 
use in thinking. Their knowledge about the previous phase of flight became less accessible, and so was not used in explaining the fault.

\section{Working Storage}

The inference processes build up the contextual overview or situation awareness in working storage. This is not the same as short-term memory, but short-term memory is an important limit to performance and is discussed first.

\section{Short-Term Memory}

Figure 6.25 shows some typical data on how much is retained in short-term memory after various time intervals. Memory decays over about $30 \mathrm{sec}$, and is worse if the person has to do another cognitive task before being tested on what the person can remember.

This memory decay is important in the design of computer-based display systems in which different display formats are called up in sequence on a screen. Suppose the user has to remember an item from one display, for use with an item on a second display. Suppose that the second display format is not familiar, so the person has to search for the second item: This search may take about $25 \mathrm{Sec}$. The first item must then be recalled after doing the cognitive

processes involved in calling up the second display and searching it. The memory data suggest that the person will have forgotten the first item on $30 \%$ of occasions.

The practical implication is that, to avoid this source of errors, it is necessary to have sufficient display area so that all the items used in any given cognitive processing can be displayed simultaneously. Minimizing non-task-related cognitive processes is a general HF/E aim, to increase processing efficiency. In this case it is also necessary in order to reduce 
errors. This requirement emphasizes the need to identify what display items are used together, in a cognitive task analysis.

\section{The Overview in Working Storage}

Although there are good reasons to argue that the cognitive processes in complex dynamic tasks build up a contextual overview of the person's present understanding and plans (Bainbridge 1993a), not much is known about this overview. This section makes some points about its capacity, its content, and the way items are stored.

Capacity. Bisseret (1970) asked air traffic area controllers, after an hour of work, what they remembered about the aircraft they had been controlling. Three groups of people were tested: trainee controllers, people who had just completed their training, and people who had worked as controllers for several years. Figure 6.26 shows the number of items recalled. The experienced controllers could remember on average 33 items. This is a much larger figure than the $7 \pm 2$ chunk capacity for static short-term memory (Miller. 1956) or the 2 items capacity of running memory for arbitrary material (Yntema \& Mueser, 1962). Evidently a person's memory capacity is improved by doing a meaningful task and by experience. A possible reason for this is given later.

Content. Bisseret also studied which items were remembered. The most frequently remembered items were flight level (33\% of items remembered), position (31\%), and time at fix (14\%). Leplat and Bisseret (1965) had previously identified the strategy the controllers used in conflict identification (checking whether aircraft are a safe distance apart). The frequency with which the items were remembered matches the sequence in which they were 
thought about: The strategy first compared aircraft flight levels, then position, then time at fix, and so on.

Sperandio (1970) studied another aspect (Fig. 6.27). He found that more items were remembered about aircraft involved in conflict than ones that were not. For nonconflict aircraft, more was remembered about aircraft that had been in radio contact. For conflict aircraft, more was remembered about aircraft on which action had been taken, and most was remembered about aircraft for which an action had been chosen but not yet made.

These results might be explained by two classic memory effects. One is the rehearsal or repetition mechanism by which items are maintained in short-term memory. The more frequently the item or aircraft has been considered by the controllers when identifying potential collisions and acting on them, the more likely it is to be remembered. The findings about aircraft in conflict could be explained by the recency effect, that items that have been rehearsed most recently are more likely to be remembered. These rehearsal and recency mechanisms make good sense as mechanisms for retaining material in real as well as in laboratory tasks.

The Form in Which Material Is Retained. The controllers studied by Bisseret (1970) remembered aircraft in pairs or threes: "There are two flying towards DIJ, one at level 180, the other below at 160," "there are two at level 150, one passed DIJ towards BRY several minutes ago, the other should arrive at X at 22," or "I've got one at level 150 which is about to pass RLP and another at level 170 which is about 10 min behind." The aircraft were not remembered by their absolute positions, but in relation to each other. Information was also remembered relative to the future; many of the errors put the aircraft too far ahead. These 
sorts of data suggest that, although rehearsal and recency are important factors, the items are not remembered simply by repeating the raw data, as in short-term memory laboratory experiments. What is remembered is the outcome of working through the strategy for comparing aircraft for potential collisions. The aircraft are remembered in terms of the key features that bring them close together-whether they are at the same level, or flying toward the same fix point, and so on.

A second anecdotal piece of evidence is that air traffic controllers talk about "losing the picture" as a whole, not piecemeal. This implies that their mental representation of the situation is an integrated structure. It is possible to suggest that experienced controllers remember more because they have better cognitive skills for recognizing the relations between aircraft, and the integrated structure makes items easier to remember.

The only problem with this integrated structure is that the understanding, predictions, and plans can form a "whole" that is so integrated and self-consistent that it becomes too strong to be changed. People may then only notice information that is consistent with their expectations, and it may be difficult to change the structure of inference if it turns out to be unsuccessful or inappropriate (this rigidity in thinking is called perceptual set).

Some Practical Implications. Some points have already been made about the importance of short-term memory in display systems. The interface also needs to be designed to support the person in developing and maintaining an overview. It is not yet known whether an overview can be obtained directly from an appropriate display, or whether the overview can only be developed by actively understanding and planning the task, with a good display enhancing this processing but not replacing it. It is important, in display systems in which the 
data needed for the whole task are not all displayed at the same time, to ensure there is a permanent overview display and that it is clear how the other possible displays are related to it.

Both control automation (replacing the human controller) and cognitive automation (replacing the human planner, diagnoser, and decision maker) can cause problems with the person's overview. A person who is expected to take over manual operation or decision making will only be able to make informed decisions about what to do after the person has built up an overview of what is happening. This may take 15-30 min to develop. The system design needs to allow for this sort of delay before a person can take over effectively (Bainbridge, 1983). Also, the data just given show that a person's ability to develop a wide overview depends on experience. This means that, to be able to take over effectively from an automated system, the person needs to practice building up this overview. Practice opportunities should therefore be allowed for in the allocation of functions between computer and person, or in other aspects of the system design such as refresher training.

\section{Planning, Multitasking, and Problem Solving}

Actions in complex dynamic tasks are not simple single units. A sequence of actions may be needed, and it may be necessary to deal with several responsibilities at the same time. Organization of behavior is an important cognitive function, which depends on and is part of the overview. This section is in three interrelated parts: planning future sequences of action; multitasking, dealing with several concurrent responsibilities, including sampling; and problem solving, devising a method of working when a suitable one is not known.

\section{Planning}


It may be more efficient to think out what to do in advance, if there is a sequence of actions to carry out, or multiple constraints to satisfy, or it would be more effective to anticipate events. Alternative actions can be considered and the optimum ones chosen, and the thinking is not done under time pressure. The planning processes may use working storage, for testing the alternatives by mental simulation, and for holding the plan as part of the overview.

In aviation, an obvious example is preflight planning. Civilian pilots plan their route in relation to predicted weather. Military pilots plan their route relative to possible dangers and the availability of evasive tactics. In high-speed, low-level flight there is not time to think out what to do during the flight, so the possibilities need to be worked out beforehand. The plan then needs to be implemented, and adjusted if changes in circumstances make this necessary. This section is in two parts, on preplanning and online revision of plans.

Preplanning. Figure 6.28 shows results from a study of preflight planning by Amalberti (1992, Expt. 2). Pilots thought out the actions to take at particular times or geographical points. Planning involves thinking about several alternative actions, and choosing the best compromise given several constraints. Some of the constraints the pilots consider are the level of risk of external events, the limits to maneuverability of the aircraft, and their level of expertise to deal with particular situations, as well as the extent to which the plan can be adapted, and what to do if circumstances mean that major changes in plan are needed.

Amalberti studied four novice pilots, who were already qualified but at the beginning of their careers, and four experts. The cognitive aims considered during planning are listed on 
the left of the figure. Each line on the right represents one pilot, and shows the sequence in which he thought about the cognitive functions. The results show that novice pilots took longer to do their planning, and that each of the novice pilots returned to reconsider at least one point he had thought about earlier. Verbal protocols collected during the planning showed that novices spent more time mentally simulating the results of proposed actions to explore their consequences. The experts did not all think about the cognitive functions in the same sequence, but only one of them reconsidered an earlier point. Their verbal protocols showed they prepared fewer responses to possible incidents than the novices.

One of the difficulties with planning is that later in planning the person may think of problems that mean that parts of the plan already devised need to be revised. Planning is an iterative process. The topics are interdependent; for example, the possibility of incidents may affect the best choice of route to or from the objective. What is chosen as the best way of meeting any one of the aims may be affected by, or affect, the best way of meeting other aims. As the topics are interdependent, there is no one optimum sequence for thinking about them. The results suggest that experts have the ability, when thinking about any one aspect of the flight, to take into account its implications for other aspects, so it does not need to be revised later.

The experts have better knowledge about the scenario, about possible incidents and levels of risk. They know more about what is likely to happen, so they need to prepare fewer alternative responses to possible incidents. The experts also know from experience the results of alternative actions, including the effects of actions on other parts of the task, so they do not need to mentally simulate making actions to check their outcomes. They also have more confidence in their own expertise to deal with given situations. All these are aspects of their 
knowledge about the general properties of the things they can do, how risky these are, how good they are at them, and so on. This meta-knowledge was introduced in the earlier section on actions, and is also central to multitasking and in workload and learning (see later discussion).

Online Adaptation of Plans. In the second part of Amalberti's study, the pilots carried out their mission plan in a high-fidelity simulator. The main flight difficulty was that they were detected by radar. The pilots responded immediately to this. The response had been been preplanned, but had to be adapted to details of the situation when it happened. The novice pilots showed much greater deviations from their original plan than the experts. Some of the young pilots slowed down before the point at which they expected to be detected, as accelerating was the only response they knew for dealing with detection. This acceleration led to a deviation from their planned course, so they found themselves in an unanticipated situation. They then made a sequence of independent, reactive, short-term decisions, because there was not time to consider the wider implications of each move. The experts made much smaller deviations from their original plan, and were able to return to the plan quickly. The reason for this was that they had not only preplanned their response to radar, they had also thought out in advance how to recover from deviations from their original plan. Again experience, and therefore training, plays a large part in effective performance.

In situations in which events happen less quickly, people may be more effective in adapting their plans to changing events at the time. The best model for the way that people adapt their plans to present circumstances is probably the opportunistic planning model of Hayes-Roth and Hayes-Roth (1979; see also Hoc, 1988). 


\section{Multitasking}

If a person has several concurrent responsibilities, each of which involves a sequence of activities, then interleaving these sequences is called multitasking. Doing this involves an extension of the processes mentioned under planning. Multitasking involves working out in advance what to do, combined with opportunistic response to events and circumstances at the time.

Examples of Multitasking. Amalberti (1992, Expt. 1) studied military pilots during simulated flight. Figure 6.29 shows part of his analysis, of activities during descent to lowlevel flight. The bottom line in this figure is a time line. The top part of the figure describes the task as a hierarchy of task goals and subgoals. The parallel double-headed arrows beneath represent the time that the pilot spent on each of the activities. These arrows are arranged in five parallel lines that represent the five main tasks in this phase of flight: maintain engine efficiency at minimum speed; control angle of descent; control heading; deal with air traffic control; and prepare for the next phase of flight. Other principal tasks that occurred in other phases of flight were: keep to planned timing of maneuvers; control turns; check safety. Figure 6.29 shows how the pilot allocated his time between the different tasks. Sometimes it is possible to meet two goals with one activity. The pilot does not necessarily complete one subtask before changing to another. Indeed, this is not often not possible in a control task, in which states and events develop over time. Usually the pilot does one thing at a time. However, it is possible for him to do two tasks together when they use different cognitive processing resources. For example, controlling descent, which uses eyes + motor coordination, can be done at the same time as communicating with air traffic control, which uses hearing + speech (see also later discussion on workload). 
Some multitasking examples are difficult to describe in a single figure. For example, Reinartz (1989), studying a team of three nuclear power plant operators, found they might work on 9 to 10 different goals at the same time. Other features of multitasking have been observed by Benson (1990):

- Multitasking may be planned ahead (a process operator studied by Beishon, 1974, made plans for up to $1.5 \mathrm{hr}$ ahead). These plans are likely to be partial, and incomplete in terms of timing and detail. Planned changes in activity may be triggered by times or events. When tasks are done frequently, much of the behavior organization may be guided by habit.

- Executing the plan. Interruptions may disrupt planned activity. The preplan is incomplete, and actual execution depends on details of the situation at the time. Some tasks may be done when they are noticed in passing (Beishon, 1974, first noticed this, and called it serendipity). This is opportunistic behavior. The timing of activities of low importance may not be preplanned, but may be fitted in in spare moments. The remaining spare moments are recognized as spare time.

- Effects of probabilities and costs. In a situation that is very unpredictable, or when the cost of failure is high, people may make the least risky commitment possible. If there is a high or variable workload, people may plan to avoid increasing their workload, and use different strategies in different workload conditions (see later discussion on workload).

A Possible Mechanism. Sampling is a simple example of multitasking in which people have to monitor several displays to keep track of changes on them. Mathematical sampling theory has been used as a model for human attention in these tasks. In the sampling 
model, the frequency of attending to an information source is related to the frequency of changes on that source. This can be a useful model of how people allocate their attention when changes to be monitored are random, as in straight and level flight, but this model is not sufficient to account for switches in behavior in more complex phases of flight.

Amalberti (1992) made some observations about switching from one task to another. He found that:

- Before changing to a different principal task the pilots review the normality of the situation, by checking that various types of redundant information are compatible with each other.

- Before starting a task that will take some time, they ensure that they are in a safe mode of flight. For example, before analyzing the radar display, they check that they are in the appropriate mode of automatic pilot.

- While waiting for feedback about one part of the task, pilots do part of another task that they know is short enough to fit into the waiting time.

- When doing high-risk, high-workload tasks, pilots are less likely to change to another task.

These findings suggest that, at the end of a subsection of a principal task, the pilots check that everything is all right They then decide (not necessarily consciously) what next to devote effort to, by combining their preplan with meta-knowledge about the alternative tasks, such as how urgent they are, how safe or predictable they are, how difficult they are, how much workload they involve, and how long they take (see later discussion on workload). 
Practical Implications. Multitasking can be preplanned, and involves metaknowledge about alternative behaviors. Both planning and knowledge develop with experience, which underlines the importance of practice and training.

The nature of multitasking also emphasizes the difficulties that could be caused by task-specific displays. If a separate display was used for each of the tasks combined in multitasking, then the user would have to call up a different display, and perhaps change coding vocabularies, each time the person changed to a different main task. This would require extra cognitive processing and extra memory load, and could make it difficult to build up an overview of the tasks considered together. This suggests an extension to the point made in the section on working storage. All the information used in all the principal tasks that may be interleaved in multitasking needs to be available at the same time, and easily crossreferenced. If this information is not available, then coordination and opportunistic behavior may not be possible.

\section{Problem Solving}

A task is familiar to a person who knows the appropriate working methods, plus the associated reference knowledge about the states that can occur, the constraints on allowed behavior, and the scenarios, mental models, and so on that describe the environmental possibilities within which the working methods must be used.

Problem solving is the general term for the cognitive processes a person uses in an unfamiliar situation, which the person does not already have an adequate working method or reference knowledge for dealing with. Planning and multitasking are also types of processing that are able to deal with situations that are not the same each time. However, both take 
existing working methods as their starting point, and either think about them as applied to the future, or work out how to interleave the working methods used for more than one task. In problem solving, a new working method is needed.

There are several ways of devising a new working method. Some are less formal techniques that do not use much cognitive processing, such as trial and error, or asking for help. There are also techniques that should not need much creativity, such as reading an instruction book. People may otherwise use one of three techniques for suggesting a new working method. Each of these uses working methods recursively; it uses a general working method to build up a specific working method.

1. Categorization. This involves grouping the problem situation with similar situations for which a working method is available. The working method that applies to this category of situation can then be used. This method is also called recognition-primed decision making. The nature of "similarity" and the decisions involved are discussed by Klein (1989).

2. Case-based reasoning. This involves thinking of a known event (a case) that is similar or analogous to the present one, and adapting the method used then for use in the present situation. This is the reason why stories about unusual events circulate within an industry. They provide people in the industry with exemplars for what they could do themselves if a similar situation arose, or with opportunities to think out for themselves what would be a better solution.

3. Reasoning from basic principles. In the psychological literature, the term problem solving may be restricted to a particular type of reasoning in which a person devises a new method of working by building it up from individual components 
(e.g., Eysenck \& Keane, 1990, chapters 11 and 12). This type of processing may be called knowledge based by some people.

A general problem-solving strategy consists of a set of general cognitive functions that have much in common with the basic cognitive functions in complex dynamic tasks (see introduction to this section). Problem solving, for example, could involve understanding the problem situation, defining what would be an acceptable solution, and identifying what facilities are available. Meeting each of these cognitive needs can be difficult, because the components need to be chosen for their appropriateness to the situation and then fitted together. This choice could involve: identifying what properties are needed from the behavior; searching for components of behavior that have the right properties (according to the meta-knowledge which the person has about them); and then combining them into a sequence.

The final step in developing a new working method is to test it, either by mental simulation, or by trial and error. This mental simulation could be similar to the techniques used in planning and multitasking. Working storage may thus be used in problem solving in two ways: to hold both the working method for building up a working method and the proposed new method; and to simulate carrying out the proposed working method to test whether it's processing requirements and outputs are acceptable.

\section{Knowledge}

Knowledge is closely involved in all modes of cognitive processing. It provides the probabilities, utilities, and alternatives considered in decision making, and the translations used in recoding. In complex tasks it provides the working methods and reference knowledge 
used in thinking about cognitive functions, and the meta-knowledge. Different strategies may use different types of reference knowledge. For example, a strategy for diagnosing faults by searching the physical structure of the device uses one type of knowledge, whereas a strategy that relates symptoms to the functional structure of the device uses another. The reference knowledge may include scenarios, categories, cases, mental models, performance criteria, and other knowledge about the device the person is working with. Some knowledge may be used mainly for answering questions, for explaining why events occur or actions are needed. This basic knowledge may also be used in problem solving.

There are many interesting fundamental questions about how these different aspects of knowledge are structured, interrelated, and accessed (Bainbridge, 1993c), but these issues are not central to this chapter. The main questions here are the relation between the type of knowledge and how it can best be displayed, and what might be an optimum general display format.

Knowledge and Representation. Any display for a complex task can show only a subset of what could be represented. Ideally, the display should make explicit the points that are important for a particular purpose, and provide a framework for thinking. The question of which display format is best for representing what aspect of knowledge has not yet been thoroughly studied, and most of the recommendations about this are assumptions based on experience (Bainbridge, 1988). For example, the folling formats are often found:

Aspect of knowledge

Geographical position

Topology, physical structure
Form of display representation

Map

Mimic/schematic, wiring diagram 
Cause-effect, functional structure

Task goals-means structure

Sequence of events or activities

Analogue variable values and limits

Evolution of changes over time
Cause-effect network, mass-flow diagram

Hierarchy

Flow diagram

Scale + pointer display

Chart recording

Each of these aspects of knowledge might occur at several levels of detail, for example, in components, subsystems, systems, and the complete device. And knowledge can be at several levels of distance from direct relevance; for example, it could be about a specific aircraft, about all aircraft of this model, about aircraft in general, about aerodynamics, or about physics.

Knowledge-display recommendations raise three sorts of question. One arises because each aspect of knowledge is one possible "slice" from the whole body of knowledge. All the types of knowledge are interrelated, but there is not a simple one-to-one relation between them. Figure 6.30 illustrates some links between different aspects of knowledge. Any strategy is unlikely to use only one type of knowledge, or to have no implications for aspects of thinking that use other types of knowledge. It might mislead the user to show different aspects of knowledge with different and separate displays that are difficult to cross-refer between, as this might restrict the thinking about the task. Knowledge about cross-links is difficult to display, and is gained by experience. This emphasizes training.

A second question is concerned with salience. Visual displays emphasise (make more salient) the aspects which can easily be represented visually. (For example, see the discussion at the end of this chapter on the limitations of Figs. 6.22 and 6.24 as models of behavior.) It 
might be unwise to make some aspects of knowledge easy to take in simply because they are easier to display, rather than because they are important in the task. There are vital types of knowledge that are not easy to display visually, such as the associations used in recoding, or the categories, cases, scenarios, and meta-knowledge used in complex thinking. These are all learned by experience. The main approach to supporting nonvisual knowledge is to provide the user with reminder lists about the alternatives (see later discussion on cued recall). Display design and training are interdependent, as they are each effective at providing different types of knowledge. It could be useful to develop task analysis techniques that identify different aspects of knowledge, as well as to do more research on how types of knowledge, and the links between them, can best be presented.

The third issue about all these multiple possible display formats repeats the questions raised previously about efficient use of codes. If a user was given all the possible display types just listed, each of which would use different codes, possibly with different display formats using the same code with different meanings (e.g., a network with nodes could be used to represent physical, functional, or hierarchical relations between items), the different codes might add to the user's difficulties in making cross connections between different aspects of knowledge.

An Optimum Format? These issues suggest the question: Is there one or a small number of formats that subsume or suggest the others? This is a question that has not yet been much studied. A pilot study (Brennan, 1987) asked people to explain an event, given either a mimic or a cause-effect diagram of the physical device involved. The people tested either did or did not already know how the device worked. The results suggested that people who did not know how the device worked were most helped by a cause-effect representation 
(which does show how it worked), whereas experts were best with the mimic representation. Contextual cues can greatly aid memory performance (e.g., Eysenck \& Keane, 1990, chapter 6). A cue is an aid to accessing the items to be recalled. The reason for expert performance with mimic displays might be that the icons and flow links on this type of display not only give direct evidence about the physical structure of the device, but they also act as cues or reminders about other knowledge the person has about the device - they evoke other parts of the scenario. This is an example from only one type of cognitive task, but it does point to the potential use of contextual cued recall in simplifying display systems. However, cued recall can only be effective with experienced people, who can recognize the cues and know what they evoke.

\section{MENTAL WORKLOAD, LEARNING, ERRORS}

Workload, learning, and errors are all aspects of the efficiency of cognitive processing. There are limits to human processing capacities, but these are difficult to define, because of the adaptability of human behavior. As a result of learning, processing becomes more efficient and adapted to what is required. As efficiency increases, mental workload may decrease. Error rates can be affected by both expertise and workload, and errors are closely involved in the processes of learning. There is a huge wealth of material that could be discussed, so the aim here is only to give a brief survey.

\section{Mental Workload}

There are a large number of issues involved in accounting for mental workload and how it is affected by different aspects of a task. This section mentions three main topics: whether people can only do one task at a time; factors affecting processing capacity; and the ways in which people typically respond to overload. 


\section{Single- or Multichannel Processing}

Many types of evidence, including the example of multitasking' in Fig. 6.29, show that people usually do one task at a time. This section looks at how people attend to one source of stimuli among many, and under what circumstances people can do more than one task at a time. As usual, the findings show how adaptable human beings are, and that there is not yet a full account of the processes involved.

Focused Attention. People have the ability to pick out one message against a background of others, visual or auditory. Studies show, however, that a person does not only process one of the stimulus sources, but takes in enough about the other possible signals to be able to separate them. This chapter has already used the notion of depth of processing, as in discrimination, recoding, sequences of recoding, and building up an overview. This notion is also involved here. Separation of two signal sources requires the least processing if they can be discriminated by physical cues, such as listening to a high voice while a low voice also speaks, or reading red lettering against a background of green letters. The sorts of factors discussed earlier on discrimination affect how easy it is to do this separation. If stimuli cannot be distinguished by physical cues, then "deeper" processing may be involved. For example, Gray and Wedderburn (1960) found that messages presented to the ears as:

$\begin{array}{lllll}\text { Left ear } & : & \text { mice } & 5 & \text { cheese } \\ \text { Right ear } & : & 3 & \text { eat } & 4 \\ \text { Were heard as } & : & (354) & & \text { (mice eat cheese) }\end{array}$


In this case, the words may be grouped by recognizing their semantic category. In some tasks deeper processing for meaning may be needed, that is, building up an overview, as in:

It is important that the subject man be car pushed house slightly boy beyond hat his shoe normal candy limits horse of tree competence pen for be only in phone this cow way book can hot one tape be pin certain stand that snaps he with is his paying teeth attention in to the the empty relevant air task and hat minimal shoe attention candy to horse the tree second or peripheral task.

(Lindsay \& Norman, 1972)

Note that if the cue used becomes ineffective, this is disconcerting. It then takes time, and a search for clues about what would be effective, before the person can orient to a new cue and continue with the task. There is also an interplay of depths of processing: When the physical cue becomes inadequate for following the message, then the reader uses continuity of meaning as a basis for finding a new physical cue. This account fits in with several points made earlier. The person is using active attention for what the person wants to take in, not passive reception of signals. The task setting provides the cue that can be used to minimize the effort needed to distinguish between signal sources. This cue than acts as a perceptual frame for searching for relevant inputs.

The concept of depth of processing was first introduced by Craik and Lockhart (1972) to explain results in some memory experiments. The word depth is here distinguished from depth in the organization of behavior, as in goal/subgoal and so on. 
Parallel Processing. The criteria defining whether or not people are able to do two tasks at the same time have so far proved elusive to identify. Figure 6.16 shows that, after high levels of practice, choice time is not affected by number of alternatives. Such tasks are said to be automated, or to require no conscious attention. They can be done at the same time as something else, unless both tasks use the same peripheral resources such as vision or hand movement. Wickens (e.g., 1984) did a series of studies showing that people can use different peripheral resources at the same time. People can also learn to do some motor tasks so that movements are monitored by feel rather than visually; then movements can be made at the same time as looking at or thinking about something else. In practice the possibility of multiple processing means that care is needed in designing tasks. One might, for example, think it would reduce unnecessary effort for an air traffic controller to have the flight strips printed out, rather than expecting the controller to write the strips by hand. However if the controller, while writing, is simultaneously thinking out how the information fits into their overview, then printing the flight strips might deprive him or her of useful attention and thinking time.

Whether or not two tasks that both involve "central" processing can be done at the same time is less clear. This is partly because what is meant by central processing has not been clearly defined. People can do two tasks at the same time if the tasks are processed by different areas of the brain-for example, a music task and a language task (Allport, Antonis, \& Reynolds, 1972) — though both tasks need to be simple and perhaps done by recoding. Going to "deeper" levels of processing, there does seem to be a limit to the extent to which people can build up distinct overviews for two different tasks at the same time. Whether or not an overview is needed to do a task may be part of the question. For example, people playing multiple chess games may have very good pattern recognition skills and so react to 
each game by recognition-primed decisions as they return to it, rather than having to keep in mind a separate and continuing overview for each of the games they are playing. Most experienced drivers can drive and hold a conversation on a different topic at the same time when the driving task is simple, but they stop talking when the driving task becomes more difficult.

This is an area in which it is challenging to identify the limits to performance, and it is probably beyond the competence of $\mathrm{HF} / \mathrm{E}$ at the moment either to define the concepts or to investigate and measure the processing involved. Fortunately, in practice the issue can often be simplified. When predicting performance, the conservative strategy is to assume that people cannot do two tasks at the same time. This will always be the worst-case performance.

\section{Factors Influencing Processing Capacity}

The amount of mental work a person can do in a given time is not a simple quantity to specify. If it is assumed that a person can only do one thing at a time, then every factor that increases the time taken to do a unit task will decrease the number of those tasks that can be done in a given time interval, and so decrease performance capacity. Thus every factor in interface design might affect performance capacity.

Focusing on performance time emphasizes performance measures of workload effects. Other important measures of workload are physiological, such as the rate of secretion of stress chemicals, and subjective, such as moods and attitudes. Any factor could be considered a "stressor" if its effect is that performance levels, stress hormone secretion rates, or subjective feelings deteriorate. The approach in this section is to indicate some key general topics, rather than to attempt a full review. There are reviews of workload topics in the 
chapters on fatigue and biological rhythms, pilot performance, and controller performance (chapters 10, 13, and 19).

The points made here are concerned with the capacities of different mental processes; extrinsic and intrinsic stressors; individual differences; and practical implications.

Capacities of Different Cognitive Resources. Different aspects of cognitive processing have different capacities. For a review of processing limits see Sage (1981). The capacity of different processes may be affected differently by different factors. Figure 6.31 shows time-of-day effects on performance in four tasks: serial search, verbal reasoning (working memory) speed, immediate retention, and alertness. The different performance trends in these tasks suggest that each task uses a different cognitive resource that responds differently to this stress. It is difficult to make reliable analyses of these differences, but some other tasks in which performance may differ in this way are coding and syllogisms (Folkhard, (1990).

Extrinsic and Intrinsic Stressors. Extrinsic stressors are stressors that apply to any person working in a particular environment, whatever task they are doing. Time-of-day, as in Fig. 6.31, is extrinsic in this sense. Some other extrinsic stressors that can affect performance capacity are noise, temperature, vibration, fumes, fatigue, and organizational culture.

Intrinsic stressors are factors that are local to a particular task. All the HF/E factors that affect performance speed or accuracy come in this category. The effect of task difficulty interacts with motivation. Easy tasks may be done better with high motivation, whereas difficult tasks are done better at lower levels of motivation. This can be explained by 
assuming that stressors affect a person's "arousal" level, and that there is an inverted-U relation between arousal level and performance (see Fig. 6.32).

Measures of stress hormones and of workforce attitudes show that several factors to do with the pacing of work, and the amount of control over their work that a person feels he or she has, can be stressors (e.g., Johansson, Aronsson, \& Lindströrn, 1978). Such aspects are of more concern in repetitive manufacturing jobs than in work such as flying or air traffic control.

Individual Differences. Individual differences affect a person's capacity for a task, and the person's willingness to do it. Aspects of individual differences fall into at least five groups.

1. Personality. Many personality dimensions, such as extroversion/introversion, sensitivity to stimuli, need for achievement or fear of success, and preference for facts/ideas or for regularity /flexibility, can affect a person's response to a particular task.

2. Interests and values. A person's interests and values affect the response to various factors in the task and the organizational climate, which influence willingness and commitment to do or learn a given task. People differ in their response to incentives or disincentives such as money, status, or transfer to a job that does not use their skills.

3. Talent Different people have different primary senses, different cognitive styles, and different basic performance abilities (e.g., Fleishman, 1975). For example, very few of us have the ability to fly high-speed aircraft. 
4. Experience. The rest of us may be able to develop higher levels of performance though practice. Even the few who can fly high-speed aircraft have millions spent on their training. The effects of training on cognitive capacities are discussed more in the section on learning.

5. Nonwork stressors. There may be nonwork stressors on an individual that affect the person's ability to cope with work, such as illness, drugs, or home problems.

Practical Implications. There are so many factors affecting the amount of effort any particular individual is able or willing to devote to a particular task at a particular time, that performance prediction might seem impossible. Actually the practical ways of dealing with this variety are familiar. There are two groups of issues, in HF/E design and in performance prediction.

Nearly all HF/E design recommendations are based on measures of performance capacity. Any factor that has a significant effect on performance should be improved, as far as is economically justifiable. Design recommendations could be made about all the intrinsic and extrinsic factors mentioned earlier, and individual differences might be considered in selection.

However, it is easier to predict that a design change will improve performance than to predict the size of the improvement. Numerical performance predictions may be made in order to assess whether a task can be done in the time available, or with the available people, or to identify limits to speed or accuracy on which design investment should best be concentrated. Obviously it is not practical to include all the possible effective factors when making such predictions. Three simplifying factors can reduce the problem. One is that, 
although smaller performance changes may give important clues about how to optimize design, from the point of view of performance prediction these factors may only be important if they make an order of amplitude difference to performance. Unfortunately, our data relevant to this issue are far from complete. The second point is that only conservative performance predictions are needed. For these purposes it may be valid to extrapolate from performance in simple laboratory tasks in which people with no relevant expertise react to random signals, which is the worst case. To predict minimum levels of performance, it may not be necessary to include the ways in which performance can improve when experienced people do tasks in which they know the redundancies, can anticipate, and so forth. The third point is that, in practice, many of the techniques for performance prediction that have been devised have the modest aim of matching expert judgements about human performance in a technique that can be used by someone less expert, rather than attempting high levels of accuracy or validity.

\section{Response to Overload}

If people doing a simple task have too much to do, they only have the options of omitting parts of the task or of accepting a lower level of accuracy in return for higher speed (Fig. 6.18). People doing more complex tasks may have more scope for responding to increased workload while maintaining acceptable task performance. This section discusses increasing efficiency, changing strategy, and practical implications.

Increasing Efficiency. Complex tasks often offer the possibility of increasing the efficiency with which a task is done. For example, Sperandio (1972) studied the radio messages of air traffic approach controllers. He found that when they were controlling (one 
aircraft they spent $18 \%$ of their time in radio communication. When there were nine aircraft, they spent $87 \%$ of their time on the radio. In simple models of mental workload:

Total workload $=$ workload in one task $\times$ number of tasks

Evidently that does not apply here, or the controllers would spend $162 \%$ of their time on the radio. Sperandio found that the controllers increased the efficiency of their radio messages in several ways. There were fewer pauses between messages. Redundant and unimportant information were omitted. And conversations were more efficient: The average number of conversations per aircraft decreased but the average number of messages per conversation increased, so fewer starting and ending procedures were necessary.

Changing Strategy. The controllers studied by Sperandio (1972) not only altered the efficiency of their messages; the message content also altered. The controllers used two strategies for bringing aircraft into the airport (this is a simplification so the description can be brief). One strategy was to treat each aircraft individually. The other was to standardize the treatment of aircraft by sending them all to a stack at a navigation fix point, from which they could all enter the airport in the same way. When using the individual strategy, the controllers asked an aircraft about its height, speed, and heading. In the standard strategy they more often told an aircraft what height and heading to use. The standard strategy required less cognitive processing for each aircraft. Sperandio found that the controllers changed from using only the individual strategy when there were three or fewer aircraft, to using only the standard strategy when there were eight or more aircraft. Expert controllers changed to the standard strategy at lower levels of workload. Sperandio argued that the controllers change to 
a strategy which requires less cognitive processing, in order to keep the total amount of cognitive processing within achievable limits (Fig 6.33A).

The relation between task performance and workload is therefore not the same in mental work as it is in physical work. In physical work, conservation of energy ensures there is a monotonic relation between physical work and task performance. In mental workload, if there an' alternative working methods for meeting given task demands, there is not necessarily a linear relation between the task performance achieved and the amount of mental work needed to achieve it. By using different methods, the same amount of mental effort can achieve different amounts of task performance (Fig. 6.33B).

In choosing an optimum working method, two adaptations are involved. A person must choose a method that meets the task demands. The person must also choose a method that maintains mental workload at an acceptable level. Whichever method is chosen will affect both the task performance achieved and the mental workload experienced (Fig. 6.34). There needs to be a mechanism for this adaptive choice of working method. This is another contextual effect that could be based on meta-knowledge. Suppose that the person knows, for each method, both how well it meets various task demands and what mental workload demands it poses. The person could then compare this meta-knowledge with the demands of the task and mental context, to choose the best method for the circumstances (Bainbridge, 1978).

Practical Implications. This flexibility of working method has several practical implications. It is not surprising that many studies have found no correlation between task performance and subjective experience of mental workload. There are also problems with 
predicting mental workload, similar to the problems of predicting performance capacity mentioned earlier.

A person can only use several alternative working methods if the performance criteria do not strictly constrain what method must be used. For example, in air traffic control, safety has much higher priority than the costs of operating the aircraft. Task analysis could check that alternative methods are possible, and perhaps what these methods are (it may not be possible to predefine all methods; see earlier discussion on problem solving and later discussion on learning).

Adaptive use of working methods suggests that strategy specific displays should not be provided, as they could remove the possibility of this flexibility for dealing with varying levels of workload. It could also be useful to train people to be aware of alternative methods and of the use of meta-knowledge in choosing between them,

When decision support systems are introduced with the aim of reducing workload. It is necessary to consider a wider situation. Decision support systems can increase rather than decrease mental workload if the user does not trust the decision support system and so frequently checks what it is doing (Moray, Hiskes, Lee, \& Muir, 1995).

\section{Learning}

Learning is another potentially huge topic. All the expertise of psychology on learning, of HF/E on training, and of educational psychology on teaching cognitive skills and knowledge could be included. As this chapter focuses on cognitive processes, this section primarily discusses cognitive skill and knowledge. The coverage only attempts a brief mention of some 
key topics, which indicate how learning interrelates with other aspects of cognitive processing rather than being a separate phase of performance.

This section uses the word skill in the sense in which it is used in psychology and in British industry. There are two key features of skilled behavior in this sense. Processing can be done with increased efficiency, either because special task-related abilities have been developed that would not be expected from the average person, or because no unnecessary movements or cognitive processing are used. And behavior is adapted to the circumstances. Choices, about what best to do next and how to do it, are adapted to the task and personal context. In this general sense, any type of behavior and any mode of cognitive processing can be skilled, so it can be confusing to use the word skill as the name for one mode of processing.

This section has three main parts: changes in behavior with experience; learning processes; and relations between mode of processing and appropriate training method.

\section{Changes Within a Mode of Processing}

This subsection briefly surveys the modes of processing that have formed one framework of this chapter, and indicates the ways in which each can change by introducing new aspects of processing or losing inefficient ones. This is a summary of points made before and is by no means complete. Learning can also lead to changes from one mode of processing to another, as discussed later.

Physical Movement Skills. By carrying out movements in a consistent environment, people can learn: 
- Which movement has which effect (i.e., they develop their meta-knowledge about movements; Fig. 6.20). This means they do not need to make exploratory actions, and their movements do not oscillate around the target. People can then act with increased speed, accuracy, and coordination, and can reach to the correct control or make the correct size of action without checking.

- To use kinesthetic rather than visual feedback.

- The behavior of a moving target, so its movements can be anticipated.

Changes in performance may extend over very long periods. For example, Crossman (1959) studied people doing the manually dexterous task of rolling cigars, and found that performance continued to improve until people had made about 5 million items.

Perceptual Skills. These are discriminations and integrations. People learn:

- The discriminations, groupings, and size, shape, and distance inferences to make.

- The probabilities and biases to use in decision making.

- The appropriate items to attend to.

- The eye movements needed to locate given displays,

Recoding. The person connects from one item to another by association, without intermediate reasoning. These associations may need to be learned as independent facts, or there may be some general rule underlying a group of recodings, such as "choose the control with its location opposite to the location of the display." Many people need a large number of repetitions before they can learn arbitrary associations. 
Sequence of Recodings. Two aspects of learning may be involved:

- When a sequence is the same each time, so that the output of one recoding and the input of the next recoding are consistent, then a person may learn to "chunk" these recodings together, to carry them out as a single unit without using intermediate working memory.

- When a goal/function can be met in the same way each time, then choosing a working method that is adapted to circumstances is not necessary. A previously flexible working method may then reduce to a sequence of transforms that does not include goals or choice of working method.

Familiar Working Methods. People need to learn:

- Appropriate working method(s).

- The reference knowledge needed while using each method. When this reference knowledge has been learned while using the method, then it may be accessed automatically, without having to think out explicitly what knowledge is needed in a particular situation.

- How to build up an integrated overview.

- Meta-knowledge about each working method, when is used in choosing the best method for a given context.

Planning and Multitasking. People can become more skilled in these activities. They can learn a general method for dealing with a situation, and the subsidiary skills for dealing with parts of it (Samurçay \& Rogalski, 1988). 
Developing New Working Methods. The process of developing new working methods can itself be more or less effective. Skill here lies in taking an optimum first approach to finding a new working method. There are several possible modes of processing for doing this.

1. Recognition-primed decisions. People can only make recognition primed decisions once they have learned the categories used. Several aspects of learning are involved:

- The features defining a category, and how to recognize that an instance has these features so is a member of the category.

- The members of a category, and their properties (e.g., for each category of situation, what to do in it).

- How to adapt a category method to specific circumstances.

2. Case-based reasoning. Cases (or, more distant from a particular task, analogies) provide examples as a basis for developing the knowledge or working method needed. To be able to do this, people need to know:

\section{- Cases}

- How to recognize which case is appropriate to which circumstances.

- How to adapt the method used in one case to different circumstances. 
3. Reasoning from basic principles. For this sort of reasoning, people need to have acquired an adequate base of knowledge about the task and the device(s) they are using, with associated meta-knowledge. The same type of knowledge may also be used for explaining events and actions.

\section{Learning Processes}

Little is known about how changes in processing take place. Similar processes may be involved in developing and maintaining physical and cognitive skills. This section indicates some mechanisms: repetition; meta-knowledge and feedback; independent goals-means; and changing modes of processing.

Repetition. Repetition is crucial for acquiring and maintaining skills. The key aspects are that, each time a person repeats a task, some aspects of the environment are the same as before, and knowledge of results is given. This knowledge of results has two functions: It gives information about how and how well the task was done, and it acts as a reward.

Meta-Knowledge and Feedback. As described in the section on movement execution, learning of motor skills involves learning both how to do an action and meta-knowledge about the action. Actions have associated expectations about their effect (meta-knowledge). Feedback about the actual effect provides information that can be used to refine the choice made next time (Fig. 6.20). Thus, during learning, feedback is used both to revise the present action and to revise the next action.

Choosing an action instruction on the basis of meta-knowledge is similar in process to choosing the working method used to maintain mental workload at an acceptable level. The 
choice of working method involves checking meta-knowledge about each method, to find which method has the properties best suited to the present situation. A similar process is also involved when developing a new cognitive working method: A person develops a working method, hoping (on the basis of a combination of meta-knowledge and mental simulation) that it will give the required result, and then revises the method on the basis of feedback about the actual effectiveness of what they do.

Independent Goals-Means. In coping with mental workload, and in developing cognitive processes while learning, several working methods may be used for meeting the same function /goal. Also the same behavior may be used to meet several goals. Thus, the link between goal and means must be flexible. The goal and means are independent in principle, although, after learning, particular working methods may become closely linked to particular goals. In the section on workload, the goal-means link was described as a point at which a decision between working method, is made on the basis of meta-knowledge.

It is generally the case (Sherrington 1906/1947) that behavior at one level of organization transfers information about the goal to be met, and constraints on how it should be met, to the lower levels of behavior organization by which the goal is met, but not detailed instructions about how to meet it. How to carry out the function is decided locally in the context at the time. As behavior is not dictated from above, but has local flexibility, human beings are not by nature well suited to following standardized procedures.

Changes in the Mode of Processing. Learning does not lead only to changes within a given mode of processing. A person may also change to a different mode of processing. If the task is consistent, than a person can learn to do the task in a more automatic way, that is, by 
using a simpler mode of processing. Inversely, when there is no fully developed working method or knowledge for meeting a given goal/function, then it is necessary to devise one. Thus the possibility or need for developing a simpler or more complex mode of processing depends both on a person's experience with the task and on the amount and types of regularity in the task. It may be possible through learning to change from any mode of processing to any other mode of processing, but two types of change are most typical: from more complex to simpler processing, or vice versa.

Someone may start a new task by developing a working method. But once the person has had an opportunity to learn the regularities in the task, the processing may become simpler. If the task and environment are sufficiently stable, the person may learn that making a choice between methods to meet a goal, or search for appropriate knowledge, is not necessary. In familiar stable situations, the working method may become so standardized that the person using it is not aware of goals or choices.

Alternatively, someone may start by learning parts of a task, and gradually become able to organize them together into a wider overview, or become able to choose behavior that is compatible with several cognitive functions. These changes depend on changes in processing efficiency. When someone first does a complex task, the person may start at the lowest levels of behavior organization, learning components of the task that will eventually be simple but that at first require all the person's problem-solving, attention and other processing resources. As the processing for doing these subtasks becomes simpler with learning, this releases processing capacity. This capacity can then be used for taking in larger segments of the task at the same time, so the person can learn about larger regularities in the task. 
In general any cognitive function, and any subgoal involved in meeting it, may be met by any mode of processing, depending on the person's experience with the task, and the details of the circumstances at the moment. A task can become "automated" or flexible at any level of behavior organization, depending on the repetitions or variety of situations experienced. Thus in some tasks a person may learn to do the perceptual-motor components automatically but have to rethink the task each time at a higher level, as $\mathrm{m}$ a professional person using an office computer. In other tasks, "higher" levels of behavior organization such as planning may become automated whereas lower levels remain flexible, as in driving to work by the same route every day. It is not necessarily the case that "higher" levels of behavior organization are only done by more complex modes of processing such as problem solving, or vice versa.

As any of the main cognitive functions in a task could become so standardized that they are done automatically or unconsciously, this is the origin of so-called "shortcuts" in processing. Inversely, at any moment, a change in the task situation, such as a fault, may mean that what could previously be done automatically now has no associated standard working method, so problem solving is needed to find one. At any time, or at any point in the task, there is the potential for a change in the mode of processing. So care is needed, if an interface design strategy is chosen of providing displays that support only one mode of processing.

\section{Some Training Implications}


Gagné (e.g., 1977) first suggested the concept that different modes of processing are best developed by different training methods. It is not appropriate to survey these methods here, but some general points link to the general themes of this chapter.

Simple Processes. Training for simple processes needs to:

- Maximize the similarity to the real task (the transfer validity) of discriminations, integrations, and recodings that are learned until they become automatic, by using high-fidelity simulation.

- Minimize the need for changes in mode of processing during learning, by presenting the task in a way that needs little problem solving to understand.

- Ensure that trainees retain a feeling of mastery, as part of their meta-knowledge about the task activities, by avoiding training methods in which errors are difficult to recover from, and by only increasing the difficulty of the task at a rate such that trainees continue to feel in control.

Complex Processes. Tasks that involve building up an overview and using alternative strategies need more than simple repetition if they are to be learned with least effort. The status of errors is different in learning complex tasks. In training for simple discriminations, recodings, and motor tasks, the emphasis is on minimizing the number of errors made, so that wrong responses do not get associated with the inputs. By contrast, when learning a complex task, an "error" can have positive value as a source of information about the nature and limits of the task. Thus in learning complex tasks, the emphasis should be more on exploring the possibilities without negative consequences, in order to develop a variety of working methods 
and wide knowledge of the task alternatives. Flexibility could be encouraged by giving trainees:

- Guided discovery exercises, in which the aim is to explore the task rather than to achieve given aims.

- Recovery exercises in which people practice recovering from nonoptimal actions.

- Problem solving and planning exercises, with or without real time pressures.

- Opportunities to share with other trainees the discoveries made.

- Practice with considering alternative working methods, and with assessing the criteria for choosing between them.

- Practice with thinking about alternative "hypotheses" for the best explanation of events, or the best action.

- Practice with multitasking,

- Practice with using different methods for developing working methods, and with the case examples and recognition categories used.

A feature of cognitive skill is having a knowledge base that is closely linked to the cognitive processing that uses it, so that the knowledge is appropriately organized and easy to access. This suggests that knowledge is best learned as part of doing the task, not separately.

Training as Part of System Design. This chapter has mentioned several ways in which training needs interact with the solutions chosen for other aspects of the system design:

- The need for training, and the quality of interface or procedure design, may be inversely related. 
- Skills are lost if they are not maintained by practice, so the amount of continuing training needed may be related to the extent of automation.

\section{Difficulties and Errors}

Errors occur when people are operating at the limits of modes of processing. Errors result from misuse of normally effective processes. The concept of relating error types to modes of processing was first suggested by Rasmussen (1982), although the scheme suggested here is somewhat different.

There are several points that the approach to complex tasks taken in this chapter suggests should be added to most error schemes. First, the notion of error needs to be expanded. In some simple tasks such as recoding it is possible to be wrong. But in control tasks and in complex tasks it is useful to think in terms of difficulty or lowered effectiveness, rather than focusing on being wrong. For example, Amalberti's novice pilots (Fig. 6.28) were already qualified. They completed the task; they just did it less effectively than the more experienced pilots. Thus, as a basis for supporting people doing complex tasks, it is useful to look at factors that make the task more difficult, as well as factors that slow behavior down or increase errors.

Second, many error schemes assume that task behavior can be broken down into small independent units, each of which may be right or wrong. In probabilistic risk assessment (PRA) or human reliability assessment (HRA) techniques, behavior is segmented into separate units. A probability of error is assigned to each unit, and the total probability of human error for the combined units is calculated by addition or multiplication. But this chapter has stressed that human behavior in complex tasks does not consist of independent 
units. The components of complex behavior are organized into an integrated interdependent structure. This means that, although PRA/HRA techniques are useful for practical purposes, any attempt to increase their fundamental validity while retaining an "independent units" model of behavior is doomed to failure (Hollnagel, 1993).

Third, as the processes of building up and using an overview are often not included in models of human processing, the related errors are also often not discussed, so they are the focus here. This section briefly suggests some of the ways in which performance can be weaker (e.g., see Bainbridge, 1993b).

Discriminations. Decisions made under uncertainty cannot always be right, and are more likely to be wrong if the evidence on which they are based is ambiguous or incomplete. Incorrect expectations about probabilities and incorrect biases about payoffs can also increase error rates. People make errors such as misattributing risk, importance, or urgency; ignoring a warning that is frequently a false alarm; or seeing what they expect to see. Some people when under stress refuse to make decisions invoking uncertainty.

Recodings. There are many sorts of error that can be attributed to mistranslations. Sometimes the person does not know the coding involved. People are more likely to make coding errors when they have to remember which specific code translation to use in which circumstances. Difficult codes are often ambiguous or inconsistent. The salience of some stimuli may give improper emphasis to them or to their most obvious meaning. 
Sequences. The items that need to be retained in working memory during a sequence of behavior may be forgotten within half a minute, if other task processing distracts or interrupts the rehearsal needed to remember the items.

In an overlearned sequence, monitoring/supervision of parts of the activity may be omitted. This can lead to "slips" in performance, or to rigid behavior that causes difficulties when the environment changes and adaptive behavior is needed.

Overview and Behavior Organization. There may be errors in organizing the search for information. People may only attend to part of the task information, fail to keep up-todate with changes in the environment, or look at details without taking an overall view. They may not get information which there is a cost on getting. They may only look for information that confirms their present interpretation of the situation ("confirmation bias"). In team work, people may assume without checking that another member of the team, particularly someone with higher status, has done something which needed doing.

There may also be errors in the allocation of time between tasks, which may lead to omissions or repetitions. People may react to events rather than anticipating events and how to deal with them. They may not apply available strategies in a systematic way. They may shift between subtasks, without relating them to the task as a whole ("thematic vagabonding," Doerner, 1987). They may break the task down into sub-problems in an inadequate way, or fail to devise intermediate subgoals, or they may continue to do parts of the task which they know how to do (“encystment," Doerner, 1987). Under high workloads, people may delay decisions in the hope that it will be possible to catch up later, or they may cycle through thinking about the task demands without taking any action. 
The overview influences a person's biases about what will happen and what to do about it. If the overview is incorrect, this can lead to inappropriate behavior or expectations. People who have completed a subtask, and so completed a part of their own overview, may fail to tell other members of the team about this. Once people have built up a complete and consistent overview, it may be difficult to change it when it turns out to be inadequate ("perceptual set"). The overview may also be lost completely if a person is interrupted.

Use of Knowledge. People's knowledge of all types may be incomplete or wrong, so they make incorrect inferences or anticipations. There may be problems with assumed shared knowledge in a team if team members change.

A person may have an incorrect or incomplete representation of the device they are using. For example, the person may not know the correct causalities or interactions, or may not be able to represent correctly the development of events over time. Or someone may use an inappropriate category in recognition-primed decisions or case in case-based reasoning.

Knowledge about probabilities may be incorrect, or used wrongly. People may be under- or overconfident. They may have a "halo effect," attributing the same probabilities to unrelated aspects. They may give inappropriate credence to information or instructions from people of higher status. Different social groups - for example, unions, management, and the general public - may have different views on the risks and payoffs of particular scenarios.

This list of human weaknesses should not distract from two important points. One is that people can be good at detecting their errors and recovering from them, if they are given 
an interface and training that enable them to do this. Therefore design to support recovery should be included in cognitive task analysis.

The second point is that care is needed with the attribution of responsibility for faults. Although it may be a given individual who makes an error, the responsibility for that error may be attributed elsewhere, to poor equipment or system design (training, workload, allocation of function, teamwork, organizational culture).

\section{NEUROTECHNOLOGY-DRIVEN JOINT COGNITIVE SYSTEMS}

This chapter has so far focused on the underlying human processing mechanisms and the human adaptation strategies used in to meet the challenges of human cognitive limitations in performing complex tasks in stressful situations. Traditionally, human-machine system designers have identified bottlenecks as well-known human limitations such as short-term memory and dual-task performance. This is the standard HF/E approach whose stated aim is to design interfaces to machines that mitigate the negative impact of human limitations (Wickens, 1992). This can lead to default function allocation schemes that consider the human and machine component independently when determining which agent is best suited to accomplish some individual task. However, if one considers both human and machine as required elements to solve the complex problem at hand, a joint cognitive system (Woods, Roth \& Benett, 1990) design approach emphasizes a collaborative, complementary design, rather than the more traditional comparative approach. Such an approach depends on the ability of both the human and machine to understand each other when performing complex tasks (Brezillin \& Pomeral, 1997). Until recently, complex cognitive processes were not directly measureable. However, technologies have advanced to the state where real-time, sensor-based inferences of specific cognitive processes are feasible. Neurotechnologies, as 
they are collectively called, use data from physiological and neurophysiological sensors as input to algorithms to provide meaningful measurements of cognitive state - such as working memory, mental workload, attention, executive function, or other complex cognitive processes.

The goal of a joint cognitive system is to amplify the human's cognitive capability when performing complex tasks under stress, when normally human performance degrades. Neurotechnology-based measurement of the cognitive state of the individual allows the automated computational system to adapt to best mitigate human cognitive decrements, with the overall goal of modifying and mediating human cognition in order to optimize joint performance, rather than optimizing the human or the computer alone. In a truly adaptive joint cognitive system, the computational system can adapt to the current state of the user, rather than forcing the user to adapt to the system (Schmorrow \& Kruse, 2002). A related field is neuroergonomics, an interdisciplinary approach that combines neuroscience (the study of the brain structure and function) and HF/E (the study of behavior in task environments). The focus of neuroergonomics is the study of how the brain functions during the execution of complex tasks in real domain settings (Parasuraman \& Rizzo, 2006).

Neurotechnology-driven adaptive joint cognitive systems offer the opportunity to blur the line between human and machine, to tightly couple human and machine capabilities, and to have the machine adapt to the current state of the user. This section, while not an exhaustive overview of the collective field, will discuss the implications of the ability to measure cognitive processes on human-machine system design. A full review of joint cognitive systems, or even adaptive joint cognitive system driven by measures of cognitive state, is beyond the scope of this section. However this section will briefly introduce different 
measures of cognitive state and provide examples of how measurement of underlying cognitive processes can drive adaptive joint cognitive systems in complex task domains.

\section{Measuring Cognitive State}

Neurophysiological- and physiological-based assessment of cognitive state has been captured in several different ways. Methods fall into three general areas - direct measures of the brain based on cerebral hemodynamics (blood flow) and those based on electromagnetic brain activity (Parasuraman \& Rizzo, 2006), and indirect measures that are based on nonbrain sensors. Direct sensing of blood flow measures include functional magnetic resonance imaging (fMRI), positron emission tomography (PET), transcranial Doppler sonography (TCD), and functional near-infrared (fNIR) imaging. Direct measures of brain activity include electroencephalogram (EEG), evoked-response potentials (ERPs), and magnetoencephalography (MEG). For a more detailed review of brain imaging techniques, see Cabeza \& Kingstone, 2001. Finally, indirect measures include utilizing electrocardiogram (ECG), galvanic skin response (Verwey \& Veltman, 1996), eyelid movement (Stern, Boyer, \& Schroeder, 1994; Veltman \& Gaillard, 1998; Neumann, 2002), pupil response (Beatty, 1982; Partala \& Surakka, 2003), and respiratory patterns (Porges \& Byrne, 1992; Backs \& Seljos, Veltman \& Gaillard, 1998). Some example techniques are briefly reviewed here.

EEG (through the use of cortical electrical activity from scalp electrodes) has been used extensively in the context of adaptive joint cognitive systems. It is the gold standard for providing high-resolution spatial and temporal indices of cognitive processes. Research has shown that EEG activity can be used to assess a variety of cognitive states that affect complex task performance. These include working memory (Gevins \& Smith, 2000), alertness (Makeig \& Jung, 1995), executive control (Garavan, Ross, Li, \& Stein, 2000), and 
visual information processing (Thorpe, Fize, \& Marlot, 1996). These findings point to the potential for using EEG measurements as the basis for driving adaptive joint cognitive systems that demonstrate a high degree of sensitivity and adaptability to human operators in complex task environments. For instance, researchers have used the engagement index, developed by NASA, in the context of mixed-initiative control of an automated system (Pope, Bogart, \& Bartolome, 1995). This method uses a ratio of power in common EEG frequency bands (beta / (alpha + theta)), where cognitively alert and focused is represented in beta, wakeful and relaxed in alpha, and a daydream state in theta. Thereby higher engagement index values estimate increased levels of task engagement. The efficacy of the engagement index as the basis for adaptive task allocation has been experimentally established. For instance, under manipulations of vigilance levels (Mikulka, Hadley, Freeman, \& Scerbo, 1999) and workload (Prinzel, Freeman, Scerbo, Mikulka, \& Pope, 2000), an adaptive system effectively detected states where human performance was likely to fall, and took steps to allocate tasks in a manner that raised overall task performance. The results associated with the engagement index highlighted the potential benefits of a neurophysiologically triggered adaptive automation.

Evoked response potentials (ERPs) are the electrical potential recorded from the brain's neural response to a specific event or stimuli. EEG sensors are used to record the ERPs, which are detected approximately 150 milliseconds after stimulus onset (Thorpe, Fize \& Marlot, 1996). ERPs have been utilized as a measure of the underlying cognitive processes necessary for processing and coordinating a response to task-relevant stimuli (Makeig, Westerfield, Jung, Enghoff, \& Townsend, 2002). The challenge in utilizing ERPs for realtime cognitive state detection is the fact that typically ERP response curves are constructed by averaging responses to hundreds of stimuli over time. However, recent advances in single- 
trial ERP detection, where sensor information is integrated spatially over multiple EEG sensors, rather then integrating sensor data over time, have been shown to be accurate (Parra et al., 2003; Gerson, Parra, \& Sajda, 2005; Mathan et al, 2006a). In one example of joint cognitive system design, ERPs have been utilized to identify critical targets within large image sets efficiently in a triage process (Mathan et al, 2006b).

As an example of direct blood flow measures, fNIR spectroscopy conducts functional brain studies using wavelengths of light, introduced at the scalp, to measure hemodynamic changes. This type of noninvasive optical imaging has been shown to be sensitive to neuronal activity (Gratton \& Fabiani, 2006), and has been used to assess cognitive workload (Izzetoglu et al., 2004).

There is an extensive research history of using cardiac, or electrocardiogram (ECG), measures to evaluate cognitive activity under a variety of task conditions. For instance, ECG has been used to measure heart-rate variability in the time domain to assess mental load (Kalsbeek \& Ettema, 1963), tonic heart rate to evaluate impact of continuous information processing (Wildervanck, Mulder, \& Michon, 1978), variability in the spectral domain as an index of cognitive workload (Wilson \& Eggemeier, 1991), and T-wave amplitude during math interruption task performance (Heslegrave \& Furedy, 1979).

\section{Adaptive Joint Cognitive Systems in Complex Task Domains}

Function allocation involves the distribution of work between humans and automated systems. In 1951, Paul Fitts published a list of the functions best suited to humans or machines (Duncan, 1986). Function allocation decisions have been based on this paradigm ever since: compare humans to computers and assign tasks accordingly. In order to do this 
comparison, however, all tasks have to be reduced to a common framework, usually mathematical or technology-based framework (Jordan, 1963). Consequently, function allocation decisions have been driven more by available technology than by user needs, optimal role assignments, or an understanding of the differences between human cognition and computer algorithmic logic. Often the human roles are relegated by default, namely tasks that are too technologically difficult or expensive to automate. What is needed is a flexible, complementary, rather than comparative approach toward function allocation in the context of both the design and execution stages of human-computer systems.

Figure 6.35 illustrates an adaptive joint cognitive system. The joint cognitive system is faced with task demands. The human interacts with the system to determine his or her working methods to address the task demands. Faced with same task demands, the adaptive automation adapts its choice of working methods based on a real-time assessment of the current capabilities of the individual. Rather than traditional human-machine systems, where functions are allocated between the human and the machine at the time the system was designed (and hence by the system designers), adaptive systems dynamically determine function allocation during task execution. If the cognitive state assessment shows the human cognitive capabilities degrading, the machine can adapt to mitigate effect on overall joint performance. 


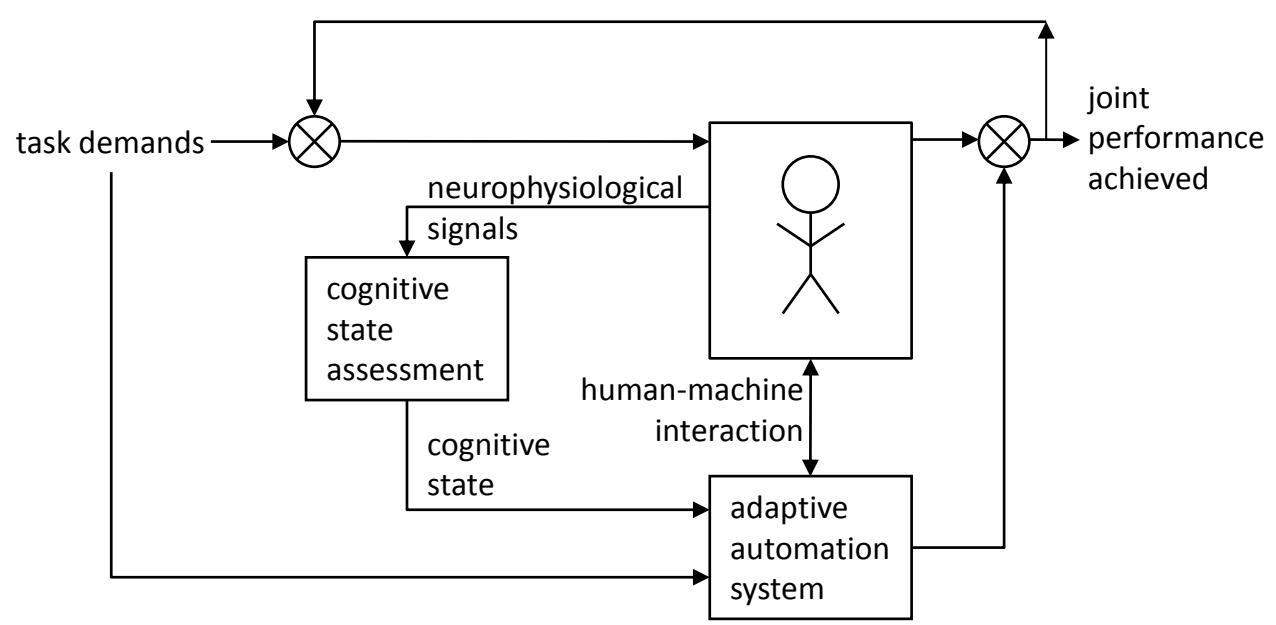

Figure 6.35. Closed loop system utilizes cognitive state feedback to adapt system function.

There are several categories of adaptation the joint cognitive system can utilize to respond to diminished human capabilities. The system, which adapts during execution in the current task environment, can either provide adaptive aiding, which makes a certain component of a task simpler, or can provide adaptive task allocation, which shifts an entire task from a larger multitask context to automation (Parasuraman, Mouloua, \& Hilburn, 1999). Adaptive joint cognitive systems must make timely decisions on how best to use varying levels of automation to support human performance. In order for an adaptive joint cognitive system to decide when to intervene, it ideally should have some model of the context of operations, be it a functional model of system performance, a model of relevant world states, and/or a model of the operator's cognitive state. The unique qualities of adaptive joint cognitive systems attempt to capitalize on the strengths and mitigate the weaknesses of a coordinated humancomputer system. By definition, their adaptive nature requires both the human and automation to take an active role in the allocation of functions. These systems require not only the human to adapt to the situation but also the automation, thereby necessitating a high degree of coordination between the human and computer in order to constantly inform the 
other about their current state. Often this coordination imposes additional tasks on the user to constantly update the system on his or her status.

Traditionally, many adaptive systems derive their inferences about the cognitive state of the operator from models, performance on the task, or from external factors related directly to the task environment (Wickens \& Hollands, 2000). Neurophysiologically and physiologically triggered adaptive joint cognitive systems offers many advantages over the more traditional approaches to human-machine system design by basing estimates of operator state on sensed data directly. These systems offer the promise of leveraging the strengths of humans and machines, augmenting human performance with automation specifically when assessed human cognitive capacity falls short of the demands imposed by task environments (Dorneich, Mathan, Ververs \& Whitlow, 2008). In addition, real-time cognitive state assessment allows the system to unobtrusively monitor state of the user, reducing or eliminating extra coordination demands placed on the user. With more refined estimates of the operator's cognitive state, measured in real-time, adaptive automation also offers the opportunity to provide aid even before the operator knows he or she is getting into trouble.

There have been a wide range of underlying cognitive processes that have been measured utilizing neurotechnology techniques, including attention, sensory memory, information processing stages, working memory, executive function, and mental workload.

Information Processing Stages. Key cognitive bottlenecks constrain information flow and the performance of decision-making, especially under stress. From an informationprocessing perspective, there is a limited amount of resources that can be applied to processing incoming information due to cognitive bottlenecks (Broadbent, 1958; Treisman, 
1964; Kahneman, 1973; Pashler, 1994). The fusion of cognitive psychology and information theory resulted in a framework — human information processing — that considers human information-processing bottlenecks in system-oriented terms such as "input, processing, output" where each stage is limited by the nature of the subsystem that executes it (Lindsay and Norman, 1977). This approach primarily considers the limitations of the human operator independently of any emergent constraints of a joint human-machine system.

With the rapid proliferation of automation within human-machine systems, researchers now conceptualize information processing stages as potential insertion points for automated aiding. For example, Parasuraman, Sheridan, and Wickens (2000) proposed that automation can be applied to four broad classes of functions that correspond to stages of human information processing: information acquisition, information analysis, decision and action selection, and action implementation, i.e. acquisition, analysis, decision, and action. This approach requires a priori, static assumptions about the relative utility of automated aiding at each stage without necessarily considering the whole system performance. In contrast, adaptive joint cognitive systems go beyond this traditional approach in several important ways. Adaptive joint cognitive systems change the task environment depending on the current state of the user, the current tasks, and the current context. Adaptive systems aim to enhance joint human-machine performance by having the system invoking varying levels of automation support in real time during task execution. Finally, adaptive automation refers to systems in which both the user and the system can initiate changes in the level of automation. (Scerbo, Freeman \& Mikulka, 2003).

Attention. Attention can be broadly defined as a mechanism for allocating cognitive and perceptual resources across controlled processes (Anderson, 1995). There are many 
varieties of attention that need to be considered to optimize their distribution (Parasuraman \& Davies, 1984): executive attention, divided attention, focused attention (both selective visual attention and selective auditory attention), and sustained attention. Breakdowns in attention lead to multiple problems: failure to notice an event in the environment, failure to distribute attention across a space, failure to switch attention to highest priority information, or failure to monitor events over a sustained period of time.

An example of an adaptive system designed to address breakdowns in attention is the Communication Scheduler (Dorneich, et al, 2005). The system utilized EEG to determine the current mental workload of the user. The Communications Scheduler scheduled and presented messages to the user based on the user's current level of mental workload, the priority of the message, and the current task context. If an incoming aural message was of higher priority than the current task, and the user's mental workload was high, the system would aggressively interrupt the user to divert their attention to the higher higher-priority message. Conversely, if the incoming message was lower priority, and mental workload was high, the message was diverted to a text display for later reading. Care must be taken when designing such a system, since inappropriate direction of attention can greatly diminish overall performance. In this case, the Communications Scheduler showed a two-fold increase in attention allocation as measured by message comprehension, with little or no decrement in secondary tasks.

Working Memory. Interactions with complex systems require humans to review available information and integrate task-relevant information in working memory in order to have an internal representation of the problem space - one that can be manipulated and analyzed to finally reach some decision (Gentner and Franks, 1983). If the user is overloaded 
with information, they expend precious cognitive resources filtering out irrelevant information which takes additional time and contributes to temporal decay of their representation in working memory (Baddeley, 1986); consequently, users are often required to make time-critical decisions based on impoverished mental models of the problem space.

An adaptive system designed to maximize working memory processes via an autonomous intelligent interruption and negotiation strategy utilized fNIR sensors to derive a diagnostic measure for verbal and spatial working memory. The system was able to dramatically increase working memory capacity for an unmanned air vehicle control task (Barker \& Richards, 2005).

Mental Workload. The previous section of this chapter described how mental workload is a function of arousal, factors affecting current processing capacity, stresses, and individual responses to overload. When a joint cognitive system's assessment of user workload is high, and additional tasks or information processing demands cannot be met, this would be a candidate time to invoke automation. However, that automation may not be appropriate when the cognitive capacity is well matched to the current task demands. Thus the adaptive system should only be invoked when the person's ability to handle the task demands breaks down. In a joint cognitive system, where automation support is provided on an as-needed basis, careful consideration must be given to the costs and benefits to determine the optimal time to invoke and disengage automation assistance.

For instance, an evaluation of an adaptive system that provided tactile navigation cues when user mental workload was overloaded demonstrated both the benefits and costs of such a system (Dorneich, Ververs, Mathan \& Whitlow, 2006). A Tactile Navigational Cueing belt 
used eight vibrating tactors in conjunction with position information from a GPS system and bearing information from a Dead Reckoning Module (DRM) to guide the participant to known waypoints. The system was only invoked when the cognitive state assessment indicated that workload was higher than available current processing capacity. Under heavy information processing demands imposed by operational tasks (such as responding to radio communications and maintaining positional awareness), and when cognitive workload exceeded capacity, automation was triggered to assist in quickly and safely navigating a complex route by temporarily alleviating the cognitive overhead of navigating to an objective, allowing available resources to be used to complete the other tasks at hand. Thus the navigation task went from being cognitively intense (involving reading a map, mental transformation from $2 \mathrm{D}$ to $3 \mathrm{D}$ space, etc) to one that is essentially a reactionary task to external stimuli that requires less cognitive resources. The overall benefit was to lower cognitive demands, allowing users to improve performance on the navigation task while not adversely affecting other tasks being done simultaneously. The potential cost of the adaptation is the loss of situation awareness of the surroundings, since the adapted task did not require attention to the environment. It is possible that the cost would never be realized (say, for instance, the user never returned to that area), but the potential cost must be weighed against the realized benefit when decided if the adaptation is triggered.

\section{Summary and Implications}

Adaptive joint cognitive systems assistance, triggered by real time classification of cognitive state, offers many advantages over traditional approaches to automation. These systems offer the promise of leveraging the strengths of humans and automation augmenting human performance with automation specifically when human abilities fall short of the demands imposed by task environments. However, by delegating critical aspects of 
complex tasks to autonomous automation components, these systems run the risk of introducing many of the problems observed in many traditional human-automation interaction contexts. The pros and cons of automating complex systems have been widely discussed in the literature (e.g. Sarter, Woods, \& Billings, 1997; Parasuraman \& Miller, 2004) However, as widely noted, poorly designed automation can have serious negative effects. Automation can relegate the operator to the status of a passive observer - serving to limit situational awareness, and induce cognitive overload when a user may be forced to inherit control from an automated system. In addition, adaption strategies that are inappropriately applied can degrade overall performance significantly. Thus the design of a joint cognitive adaptive system must explicitly considered the costs as well as the benefits of mitigation when deciding when and how to intervene in the decision making process.

\section{CONCLUSION}

There are several integrative concepts in this chapter.

Cognitive Goals. In complex tasks people use cognitive goals when implementing task goals. A person's cognitive goals are important in organizing the person's behavior, in directing attention to parts of the task, in choosing the best method for meeting a given goal, and in developing new working methods. The cognitive goals might be met in different ways in different circumstances, so the goals and the processes for meeting them can be independent. For example, flying an aircraft involves predicting the weather, and this may be done in different ways before and during the flight.

Contextual Overview. People, and to a lesser extend automated systems, build up an overview of understanding and planning, which then acts as the context for later activity. The 
overview provides data, expectations and values, and the criteria for deciding what would be the next best thing to do and how to do it.

Goal-Means Independence and Meta-Knowledge. Meta-knowledge is knowledge about knowledge, such as the likelihood of alternative explanations of what is happening, or the difficulty of carrying out a particular action. Alternative working methods have associated with them meta-knowledge about their properties. Decisions about how best to meet a particular aim are based on meta-knowledge, and are involved in adapting behavior to particular circumstances and in the control of multitasking and mental workload and in learning. Joint cognitive systems blur the line between human and machine precisely because both the human and machine employ meta-knowledge to adapt their behavior to best accomplish goals in the current context.

Modes of Processing. As well as using different working methods, people may use different modes of processing, such as knowing the answer by association or thinking out a new working method. The mode of processing used varies from moment to moment, depending on the task and the person's experience.

\section{Modeling Human Behavior}

Basing $\mathrm{HF} / \mathrm{E}$ on an analysis of behavior into small independent units fits well with a "sequential stages" concept of the underlying structure of human behavior. But a sequential stages model does not include many of the key features of complex tasks such as flying and air traffic control. Complex behavior is better described by a contextual model, in which processing builds up an overview that determines what processing is done next and how, which in turn updates the overview, and so on. In this mechanism for behavior organization, 
choices about what to do and how to do it depend on details of the immediate situation interacting with the individual's nature and previous experience. It is a tenant of joint cognitive system design that knowledge of the human cognitive state is insufficient without knowledge of the current task context in which to interpret it.

The aspects missing from many sequential stages models are:

- The goal-oriented nature of behavior, and the independence of goals from the means by which they are met.

- The continuing overview.

- The flexible sequencing of cognitive activity, and the organization of multitasking.

- The knowledge base, and the resulting predictions, anticipations, and active search for information that are part of top-down processing.

Some of these aspects require a fundamental change in the nature of the model used. The most important aspect to add is the overview, as all cognitive processes are done within the context provided by this overview, and the sequence in which they are done is determined by what is in the overview.

A simple version of a contextual model has been suggested in Figs. 6.22 and 6.24. These figures can act as an aide-mémoire about contextual processing, but any one-page representation can only indicate some features of what could be expressed. These simple figures do not make explicit important aspects such as:

- Risky decision making and the effects of biases. 
- Goal orientation of behavior.

- Typical sequences of activity.

- Different modes of processing, including devising new working methods.

- Use of meta-knowledge.

Perhaps the most important disadvantage of the one-page contextual model will be felt by people who are concerned with tasks that are entirely sequential, rather than cyclic as in flying or air traffic control. But one may argue that although dependencies may define the order in which some parts of a task are done, it could still be useful, when designing to support sequential tasks, to consider the task sequence as a frame for structuring the overt behavior, whereas the underlying order of thinking about task aspects may be more varied (cf. Fig. 6.28).

\section{The Difficulty of $\mathrm{HF} / \mathrm{E}$}

Contextual processing underlies two types of difficulty for HF/E. One group of issues is concerned with HF/E techniques. As indicated earlier, the overview suggests the need for several additions to $\mathrm{HF} / \mathrm{E}$ techniques:

- Considering the codings used in the task as a whole, rather than for isolated subtasks.

- Orienting cognitive task analysis toward the cognitive goals or functions to be met, as an intermediary between the task goals and the cognitive processing. (Analyzing either goals or working methods alone is necessary but not sufficient.)

- Designing the interface, training, and allocation of function between people and machines in a more dynamic way, to support the use of the contextual overview, 
alternative strategies, and the processes involved in the development of new working methods for both humans and machines in a joint cognitive systems design approach.

- Extending human error schemes to include difficulties with the overview and with the organization of sequences of behavior.

The second group of issues is concerned with a fundamental complexity problem in human behavior and therefore in HF/E. Human behavior is adapted to the particular circumstances in which it is done. This does not make it impossible to develop a general model of human behavior, but it does make it impossible to predict human behavior in detail. Predicting human behavior is like weather prediction: It is not possible to be right, but it is possible to be useful. Any HF/E answer is always going to be context sensitive. The continuing complaint of $\mathrm{HF} / \mathrm{E}$ practitioners, that researchers do not provide them with what they need, is a consequence of the fundamental nature of human behavior. Specific tests of what happens in specific circumstances will always be necessary. What models of human behavior can provide is, not the details, but the key issues to focus on when doing such tests or when developing and applying HF/E techniques.

\section{REFERENCES}

Allport, D. A., Antonis, B., \& Reynolds, P, (1972). On the division of attention: A disproof of the single channel hypothesis. Quarterly Journal of Experimental Psychology, 24. $225-235$.

Amalberti, R. (1992). Modèles d'activite en conduite de processus rapides: implications pour l'assistance á la conduite. Unpublished doctoral thesis, University of Paris. 
Anderson, J.R. (1995). Cognitive Psychology and its Implications (2nd Ed.). New York: Freeman.

Atkinson, W. H., Crumley, L. M., \& Willis, M P. (1952). A study of the requirements for letters, numbers and markings to be used on trans-illuminated aircraft control; panels. Part 5: the comparative legibility of three fonts for numerals (Report No. TED NAM EL-609, part 5). Naval Air Material Center, Aeronautical Medical Equipment Laboratory.

Backs, R.W. \& Seljos, K.A. (1994). Metabolic and cardiorespiratory measures of mental effort: The effects of level of difficulty in a working-memory task. International Journal of Psychophysiology, 16(1), 57-68.

Baddeley, A. (1986). Working Memory. Oxford: Clarendon Press.

Bailey, R. W. (1989). Human performance engineering. A guide for system designers (2nd (ed.). London Prentice Hall.

Bambridge, L (1974). Problems in the assessment of mental load. Le Travail Humain, 37, 279-302.

Bambridge, L. (1978). Forgotten alternatives in skill and workload. Ergonomics, 21, 169-185.

Bambridge, L. (1983). Ironies of automation. Automatica, 19, 775-779. 
Bambridge, L. (1988). Types of representation, In L. P. Goodstein, H. B. Anderson, \& S. E. Olsen (Eds.), Tasks, errors and mental models (pp. 70-91). London: Taylor \& Francis.

Bambridge, L. (1991). Multiplexed VDT display systems. In G. R. S. Weir \& J. L. Alty (Eds.), Human-computer interaction and complex systems (pp. 189-210). London: Academic Press.

Bambridge, L. (1993a). Building up behavioural complexity from a cognitive processing element (p. 95). London: Department of Psychology, University College London.

Bambridge, L. (1993b). Difficulties and errors in complex dynamic tasks. Unpublished manuscript, University College London.

Bambridge, L. (1993c). Typos of hierarchy imply types of model. Ergonomics, 36, 13991412.

Barker \& Richards, (2005). The Boeing Team Fundamentals of Augmented Cognition. In D. Schmorrow (Ed.), Foundations of Augmented Cognition. Routledge.

Barnard, P. (1987). Cognitive resources and the learning of human-computer dialogues. In J. M. Carroll (Ed.), Interfacing thought: Cognitive aspects of human-computer interaction. Cambridge, MA; MIT Press. 
Beatty, J. (1982). Task-Evoked Pupillary Responses, Processing Load, and the Structure of Processing Resources. Psychological Bulletin,_91(2), 276-292.

Beishon, R. J. (1974). An analysis and simulation of an operator's behaviour in cotrolling continuums baking ovens. In E. Edwards \& F. P. Lees (Eds). The human operator in process control (pp. 79-90). London: Taylor \& Francis.

Benson, J. M. (1990). The development of a model of multi-tasking behaviour. Unpublished doctoral thests, Ergonomics Unit, University College London.

Bisseret, A. (1970). Mémoire opérationelle et structure du travail. Bullets de Psychologic, XXIV, 280-294. English summary in Ergonomics, 14, 565-570 (1971).

Brennan, A. C. C. (1987). Cognitive support for process control: designing system representations. Unpublished master's thesis, Ergonomics Unit, University College London.

Brezillin, P. \& Pomeral, J.-C. (1997). Joint Cognitive Systems, Cooperative Systems and Decision Support Systems: A Cooperation in Context. Proc. of the European Conference on Cognitive Science, Manchester, UK, April 1997, pp. 129-139.

Briggs, G. E., \&. Rockway, M. R (1966). Learning and performance as a function of the percentage of pursuit tracking component in a tracking display. Journal of Experimental Psychology. 71. 165-169.

Broadbent, D. E. (1958). Perception and Communication. New York: Pergamon 
Cabeza, R. \& Kingstone, A. (Eds.) (2001). Handbook of Functional Neuroimaging of Cognition. Cambridge, MA: The MIT Press.

Crark, F. I. M., \& Lockhart, R. S. (1972). Levels of processing: A framework for memory research. Journal of Verbal Learning and Verbal Behaviour, 11, 671-684.

Crossman, E. R. F. W. (1959). A theory of the acquisition of speed-skill. Ergonomics. 2, $153-166$.

Crossman, E. R. F. W., \& Goodeve, P. J. (1963, April) Feedback control of hand-movement and Fitts' Law, Communication to the Experimental Psychology Society, University of Oxford.

Davis, D. R. (1966). Railway signal!' passed at danger: The drivers, circumstances and psychological processes. Ergonomics. 9, 211-222.

Doerner, D. (1987). On the difficulties people have in dealing with complexity. In J. Rasmussen, K. D. Duncan, \& J. Leplat (Eds.), New technology and human error (pp. 97-109). Chichester Wiley.

Dorneich, M.C., Whitlow, S.D., Mathan, S., Carciofini, J., \& Ververs, P.M. (2005). The Communications Scheduler: A Task Scheduling Mitigation For A Closed Loop Adaptive System. Proceedings of the 11th International Conference on Human- 
Computer Interaction, (HCI International 2005), Las Vegas, NV, USA: Lawrence Erlbaum.

Dorneich, M.C., Ververs, P.M., Mathan, S., and Whitlow, S.D, (2006). "Evaluation of a Tactile Navigation Cueing System Triggered by a Real-Time Assessment of Cognitive State," Proceedings of the Human Factors and Ergonomics Society Conference 2006, San Francisco, CA.

Dorneich, M.C., Mathan, S., Ververs, P.M, and M.C., Whitlow, S.D. (2008), "Cognitive State Estimation in Mobile Environments", Augmented Cognition: A Practitioner's Guide, Schmorrow D., \& Stanney K. (Editors), Santa Monica: HFES Press.

Duncan, J. (1986). Disorganisation of behaviour after frontal lobe damage. Cognitive Neuropychology, 3, 271-90.

Easterby, R. S. (1970). The perception of symbols for machine displays. Ergonomics, 13. $149-158$.

Ellis, A. W. (1993). Reading, writing and dyslexia: A cognitive analysis. Hillsdale NJ: Lawrence Erlbaum Associates.

Eysenck, M. W., \& Keane, M. T. (1990). Cognitive psychology: A student's handbook. Hillsdale, NJ: Lawrence Erlbaum Associates. 
Fitts, P. M. (1954). The information capacity of the human motor system in controlling the amplitude of movement. Journal of Experimental Psychology, 47, 381-391.

Fitts, P. M., \& Deininger, R. L. (1954). S-R compatibility: Correspondence among paired elements within stimulus and response codes. Journal of Experimental Psychology, 48, $483-492$.

Fitts, P. M., \& Jones, R. E. (1961a). Analysis of factors contributing to 460 "pilot error" experiences in operating aircraft controls (Memorandum Report No. TSEAA-694-12, Aero Medical Laboratory, Air Materiel Command, Wright Patterson Air Force Base, Dayton, Ohio. July 1, 1947). In H. W. Sinaiko (Ed.), Selected papers on human factors in the design and use of control system (pp, 332-358). New York: Dover. (Original work written 1947)

Fitts, P. M., \& Jones, R. E. (1961b). Psychological aspects of instrument display. 1: Analysis of 270 "pilot error" experiences in reading and interpreting aircraft instruments (Memorandum Report No. TSEAA-694-12A, Aero Medical Laboratory, Air Materiel Command. Wright Patterson Air Force Base, Dayton, Ohio. October 1, 1947), In H, W. Sinaiko (Ed,), Selected papers on human factors $m$ the design and use of control systems (pp. 359-396). New York: Dover. (Original work written 1947)

Fleishman, E. A (1975). Taxonomic problems in human performance research. In W. T. Singleton \& P. Spurgeon (Eds.), Measurement of human resources (pp. 49-72). London: Taylor \& Francis. 
Folkhard, S. (1990). Circadian performance rhythms: Some practical and theoretical implications. In D. E. Broadbent, L Reason, \& A. Baddeley (Eds.), Human factors in hazardous situations (pp. 543-553). Oxford: Clarendon Press.

Gagné. R. M. (1977). The conditions of learning (3rd ed.). New York: Holt, Rinehart \& Winston.

Gallaher, P. D., Hunt, R. A., \& Williges, R. C. (977), A regression approach to generate aircraft predictor information. Human Factors, 19, 549-555.

Garavan, H., Ross, T. J., Li, S.-J., \& Stein, E. A. (2000). A parametric manipulation of central executive functioning using fMRI. Cerebral Cortex, 10, 585-592.

Gerson, A.D., Parra, L.C., and Sajda, P. (2005) Cortical Origins of Response Time Variability during Rapid Discrimination of Visual Objects, NeuroImage, 28 (2) 326341.

Gevins, A., \& Smith, M. E. (2000). Neurophysiological measures of working memory and individual differences in cognitive ability and cognitive style. Cerebral Cortex, 10, 829-839.

Gibson, J. J. (1950). The perception of the visual world. Boston: Houghton Mifflin.

Gratton, G. \& Fabiani, M. (2006). Optical Imaging of Brain Function. In R. Parasuraman \& M. Rizzo (eds.), Neroergonomics (pp. 65-81). Oxforn: Oxford University Press. 
Gray, J. A., \& Wedderburn, A. A. I. (1960). Grouping strategies with simultaneous stimuli. Quarterly Journal of Experimental Psychology, 12, 180-184.

Green, R. (1990) Human error on the flight deck. In D. E. Broadbent, J. Reason, \& A. Baddeley (eds.), Human factors in hazardous situations (pp. 55-63). Oxford: Clarendon Press.

Grether, W. F. (1949), The design of long-scale indicators for speed and accuracy of quantitative reading, Journal of Applied Psychology, 33, 363-372.

Hayes-Roth, B., \& Hayes-Roth, F. (1979). A cognitive model of planning. Cognitive Science, $3,275-310$.

Helson, H. (1964), Adaptation-level theory. New York: Harper and Row.

Heslegrave, R.J., \& Furedy, J.J. (1979). Sensitivities of HR and T-wave amplitude for detecting cognitive and anticipatory stress. Physiology \& Behavior, 22(1), 17-23. The Netherlands: Elsevier Science.

Hoc, J.-M. (1988), Cognitive psychology of planning. London: Academic Press.

Hollnagel, E. (1993), Reliability of cognition: Foundations of human reliability analysis. London: Academic Press. 
Howland, D., \& Noble, M. (1955). The effect of physical constraints on a control on tracking performance journal of Experimental Psychology, 46, 353-360.

Hukki, K., \& Norros, L.,.(1993). Diagnostic orientation in control of disturbance situations. Ergonomics, 35, 1317-1328.

Hunt. D.P., \& Warrick, M. J., (1957). Accuracy of blind positioning of a rotary control (USAF WADC Tech. Notes 52-106). U.S. Air Force.

Izzetoglu, K., S. Bunce, Onaral B, Pourrezaei K, \& Chance B (2004). Functional optical brain imaging using near-infrared during cognitive tasks. International Journal of HumanComputer Interaction, 17(2), 211-227.

Johansson, G., Aronsson, G., \& Lindström, B. G. (1978). Social psychological and neuroendocrine stress reactions in highly mechanised work. Ergonomics, 21, 583-599.

Jordan, N. (1963). Allocation of functions between man and machines in automated systems. Journal of Applied Psychology, 47, 161-165.

Lindsay, P.H. and Norman, D.A. (1977). Human Information Processing: An Introduction to Psychology, Second Edition. Academic Press, New York.

Kahneman, D., Slovic, P., \& Tversky, A. (Eds.). (1982). Judgement under uncertainty; Heuristics and biases. New York: Cambridge University Press. 
Kahneman, D. (1973). Attention and Effort. Englewood Cliffs, NJ: Prentice-Hall.

Kalsbeek, J.W.H. \& Ettema, J.H. (1963). Scored irregularity of the heart pattern and measurement of perceptual or mental load. Ergonomics, 6, 306-307.

Klein, G. A., (1989). Recognition-primed decisions. In W. B. Rouse (Ed.), Advances in manmachine systems research (Vol. 5, pp. 47-92). Greenwich, CT: JAI Press.

Leplat, J., \& Bisseret, A. (1965). Analyse des processus de traitement de l'information chez le controleur de la navigation aerienne. Bulletin dunCERP, XIV, 51-67. English translation in Controller: IFACTCA Journal, 5, 13-22 (1965).

Lindsay, P. H., \&. Norman, D. A. (1972). Human information processing. NewYork: Academic Press.

Makeig S, Westerfield M, Jung T-P, Enghoff S, Townsend J (2002) Dynamic brain sources of visual evoked responses. Science 295: 690-693

Makeig, S., \& Jung, T -P. (1995). Changes in alertness are a principal component of variance in the EEG spectrum. NeuroReport, 7(1), 213-216.

Mathan, S., Whitlow, S., Erdogmus, D., Pavel, M., Ververs, P., Dorneich, M. (2006a) Neurophysiologically Driven Image Triage. CHI '06 extended abstracts on Human factors in computing systems. Proceedings of the 2006 Conference on Human Factors in Computing Systems. Montreal, Canada. 1085 - 1090. 
Mathan, S., Ververs, P., Dorneich, M., Whitlow, S., Carciofini, J., Erdogmus, Pavel, M., Huang, C., Lan, T., and Adami, A. (2006b), "Neurotechnology for Image Analysis: Searching For Needles in Haystacks Efficiently", Proceedings of the $2^{\text {nd }}$ Augmented Cognition International, San Francisco, CA.

Marshall, E. C., Scanlon, K. E., Shepherd, A., \& Duncan, K. D. (1981). Panel diagnosis training for major hazard continuous process installations. Chemical Engineer, 365, $66-69$

Mikulka, P., Hadley, G., Freeman, F., \& Scerbo, M. (1999). The effects of a biocybernetic system on vigilance decrement. Proceedings of the $43^{\text {rd }}$ Annual Meeting of the Human Factors and Ergonomics Society, Santa Monica, CA: HFES.

Miller, G. A., (1956). The magical number seven, plus or minus two: Some limits on our capacity for processing information. Psychological Review, 63, 81-97.

Miller, G. A., Heise, G. A., \& Lichten, W. 1951). The intelligibility of speech as a function of the context of the test materials. Journal of Experimental Psychology, 41, 329-335.

Moray, N. P., Hiskes, D., Lee, J., \& Muir, B. M., (1995). Trust and human intervention in automated systems. In J. M. Hoc, P.C. Cacciabue, \& E. Hollnagel (Eds.), Expertise and technology: Cognition and human-computer cooperation (pp. 183-194). Hillsdale, NJ: Lawrence Erlbaum Associates. 
Neumann, D.L. (2002). Effect of varying levels of mental workload on startle eyeblink modulation. Ergonomics, 45(8), 583-602.

Oborne, D (1995). Ergonomics at work ( $3^{\text {rd }}$ ed.). Chichester: Wiley.

Parasuraman, R. \& Davies, D. R. (1984). Varieties of Attention. New York: Academic Press.

Parasuraman, R., \& Miller, C. (2004). Trust and etiquette in high-criticality automated systems. Communications of the Association for Computing Machinery, 47(4), 51-55.

Parasuraman, R., Mouloua, M., \& Hilburn, B. (1999). Adaptive aiding and adaptive task allocation enhance human-machine interaction. In M.W. Scerbo \& M. Mouloua (Eds.), Automation technology and human performance: Current research and trends (pp. 129-133). Mahwah, NJ: Erlbaum.

Parasuraman, R., Sheridan, T.B., and Wickens, C.D. (2000). A model for types and levels of human interaction with automation. IEEE Transaction on Systems, Man, and Cybernetics--Part A: Systems and Humans, 30(3), 286-297.

Parasuraman, R. \& Rizzo, M. (2006). Introduction to Neroergonomics. In R. Parasuraman \& M. Rizzo (eds.), Neroergonomics (pp. 3-14). Oxforn: Oxford University Press.

Parra, L., Alvino, C., Tang, A., Pearlmutter, B., Yeung, N., Osman, A., and Sajda, P. (2003), Single Trial Detection in EEG and MEG: Keeping it Linear. Neurocomputing. (5254) $177-183$. 
Partala, T. \& Surakka, V. (2003). Pupil size variation as an indication of affective processing. International Journal of Human-Computer Studies,_59(1-2), 185-198.

Pashler, H (1994) Dual-task interference in simple tasks: data and theory. Psychological Bulletin, 116, 220-244.

Pheasant, S (1991). Ergonomics: Work and health. London: Macmillan.

Pope, A.T., Bogart, E.H., \& Bartolome, D. (1995). Biocybernetic system evaluates indices of operator engagement. Biological Psychology, 40, 187-196.

Porges, S.W. \& Byrne, E.A. (1992). Research Methods for Measurement of Heart-Rate and Respiration." Biological Psychology,_34(2-3), 93-130.

Posner, M. I., \& Rossman, E (1965). Effect of size and location of informational transforms upon short-term retention. Journal of Occupational Accidents, 4, 311-335.

Prinzel, L.J., Freeman, F.G., Scerbo, M.W., Mikulka, P.J., \& Pope, A.T. (2000). A closedloop system for examining psychophysiological measures for adaptive automation. International Journal of Aviation Psychology, 10, 393-410.

Rasmussen, J. (1982). Human errors: A taxonomy for describing human malfunction in industrial installations. Journal of Occupational Accidents, 4, 311-335. 
Reinartz, S. J. (1989). Analysis of team behaviour during simulated nuclear power plant incidents. In E. D. Megaw (Ed.), Contemporary ergonomics 1989 (pp. 188-193). London: Taylor \& Francis.

Rouse, W. B. (1980). Systems engineering models of human-machine interaction. Amsterdam: North Holland.

Rumelhart, D. E. (1977). Towards an interactive model of reading. In S. Dornic (Ed.), Attention and performance VI. Hillsdale, NJ: Lawrence Erldaum Associates.

Sage, A. P. (1981). Behavioural and organizational considerations in the design of information systems and processes for planning and decision support. IEEE Transactions on Systems, Man and Cybernetics, SMC-11 610-678.

Samurçay. R, \& Rogalski. J. (1988). Analysis of operator's cognitive activities in learning and using a method for decision making in public safety. In J. Patrick \& K. D. Duncan (Eds.), Training, human decision making and control. Amsterdam: North-Holland.

Sarter, N. B., Woods, D. D., \& Billings, C. E., (1997) "Automation surprises," Handbook of Human Factors and Ergonomics, 2nd Edition, Salvendy, Gavriel (Ed)., Wiley and Sons, 1997.

Savin, H. B., \& Perchonock, E. (1965). Grammatical structure and the immediate recall of English sentences. Journal of Verbal Learning and Verbal Behaviour, 4, 348-353. 
Scerbo, M. W.; Freeman, F. G. \& Mikulka, P. J.A brain-based system for adaptive automationTheoretical Issues in Ergonomic Science, 2003, 4, 200-219.

Schmorrow, D.D. \& Kruse, A.A. (2002). Improving human performance through advanced cognitive system technology. Proceedings of the Interservice/Industry Training, Simulation and Education Conference (I/ITSEC'02), Orlando, FL.

Shaffer. L. H. (1973). Latency mechanisms in transcription. In 5. Kornblum (Ed.), Attention and performance IV (pp. 435-446). London: Academic Press.

Sheridan, T. B., \& Ferell, W. R. (1974). Man machine systems: Information, control and decision models of human performance. Cambridge, MA: MIT Press.

Sherrington, C. (1974). The integrative action of the nervous system. Cambridge: Cambridge Universtiy Press. (Original work published 1906)

Smith, S. L., \& Thomas, D. W. (1964), Color versus shape coding in information displays. Journal of Applied Psychology, 48, 137-146.

Sperandio, J. C., (1970). Charge de travail et mémorization en contrôle d'approche (Report No. IRIA CENA, CO 7009, R24). Institut de Recherche en Informatique et Aeronautique, Paris.

Sperandio, J. C. (1972). Charge de travail et régulation des processus opératoires. Le Travail Humain, 35, 86-98. English summary in Ergonomics, 14, 571-577(1971). 
Stern, J.A., Boyer, D., \& Schroeder, D. (1994). Blink rate: A possible measure of fatigue. Human Factors, 36(2), 285-297.

Terchner, W. H., \& Krebs, M. J. (1974). Laws of visual choice reaction time. Psychological Review, 81, 75-98.

Thorpe S., Fize D., \& Marlot C. (1996). Speed of processing in the human visual system. Nature, 381, 520-522.

Treisman, A.M. (1964). Verbal cues, language, and meaning in selective attention. American Journal of Psychology, 77, 206-219

Veltman, J.A. \& Gaillard, A.W.K. (1998). Physiological workload reactions to increasing levels of task difficulty. Ergonomics, 41(5), 656-669.

Verwey, W. B. \& H. A. Veltman (1996). Detecting short periods of elevated workload: A comparison of nine workload assessment techniques. Journal of Experimental Psychology-Applied, 2(3), 270-285.

Wickens, C.D. \& Hollands, J. (2000). Engineering Psychology and Human Performance. Prentice Hall, 3rd edition 
Wickens, C.D., (1992). Engineering psychology and human performance (2nd ed). New York: Harper Collins.

Wickens, C. D. (1984). Processing resources in attention. In R. Parasuraman \&. D. R. Davies (Eds.), Varieties of attention. London: Academic Press.

Wildervanck, C., Mulder, G. \& Michon, J.A. (1978). Mapping mental load in car driving. Ergonomics, 21, 225-229.

Wilson, J. R., \& Corlett, E. N. (1995). Evaluation of human work: A practical ergonomics methodology (2nd ed.). London: Taylor \& Francis.

Wilson, G. F., \& Eggemeier, F. T. (1991). Physiological measures of workload in multi-task environ-ments. In D. Damos (Ed.), Multiple-task performance (pp. 329-360). London: Taylor \& Francis.

Winograd, T. (1972). Understanding natural language. Edinburgh: Edinburgh University Press. Reprinted from Cognitive Psychology, 1972, 3, 1-195.

Woods, D.D., Roth, E.M., \& Benett, K. (1990). Explorations in joint human-machine cognitive systems. In Robertson S. Zachary W. \& Black J.B. (Eds.) Cognition, Computing and Cooperation. Ablex (pp. 123-158).

Yntema, D. B., \& Mueser, G, E. (1962). Keeping track of variables that have few or many states. Journal of Experimental Psychology, 63. 391-395. 
FIG. 6.1. Increasing sensitivity to light after time in darkness (“dark adaptation").

FIG. 6.2. The sensitivity of the eye when adapted to three different levels of average illumination. At each adaptation level, the eye is good at discriminating between intensities around that level.

FIG. 6.3. Knowledge about the occurrence of intensities. Decision making uses knowledge about the alternatives, based on previous experience.

FIG. 6.4. An example of change in the bias used in decision making. If rewarded for "hits," bias changes to maximize payoff ("false alarms" also increase).

FIG. 6.5 Gestalt grouping processes relate together the elements of a predictor landing display. (Reprinted with permission from Human Factors. Vol. 19, No. 6, 1977. Copyright (C) 1977 by the Human Factors and Ergonomics Society. All rights reserved).

FIG. 6.6. Shape and size "constancy": the same cube with the same ellipse in three different positions. The ellipses are computer-generated duplicates.

FIG. 6.7. Ambiguous "wife/mother-in-law" figure. The same stimulus can be given different interpretations.

FIG. 6.8. Percentage of words heard correctly in noise, as a function of the number of different words that might occur (Miller, Heise, \& Lichten, 1951) 
FIG. 6.9. Reading errors with three different digit designs (Atkinson, Crumley, \& Willis, 1952). Errors are fewest with the design which minimizes the number of elements which the alternatives have in common.

FIG. 6.10 "Iconic" display: Eight variables are displayed, measured outward from the center. When all eight variables are on target, the display has an octagon shape.

FIG. 6.11. Symbols used in discrimination tests (Smith \& Thomas, 1964; copyright (C) 1964 by the American Psychological Association. Reproduced with permission).

FIG. 6.12. Effect of relative spatial layout of signals and responses on response time (fitts \& Deininger, 1954).

FIG. 6.13. Speed and accuracy of reading different altimeter designs (Grether, 1949).

FIG. 6.14. Graph of pilot data in Fig. 6.13.

FIG. 6.15. Two designs for the attitude indicator, showing incompatible movements.

FIG. 6.16. Response times are affected by the number of alternatives to be responded to, the nature of the "code" linking the signal and response, and the amount of practice (Teichner \& Krebs, 1974; Copyright (C) 1974 by the American Psychological Association. Reproduced with permission). 
FIG. 6.17. Effect of preview, and predictability of material, on response time (based on data in Shaffer, 1973; Copyright (C) 1973 Academic Press. Reproduced with permission).

FIG. 6.18. Speed-accuracy tradeoff in two-choice reactions, and the effect of stimulusresponse compatibility.

FIG. 6.19. Execution of movements of different sizes (Crossman \& Goodeve, 1963).

FIG. 6.20. Double use of feedback in learning to make movements.

FIG. 6.21. Errors in tracking performance using pursuit and compensatory displays (Briggs \& Rockway, 1966; copyright (C) 1966 by the American Psychological Association. Reproduced with permission).

FIG. 6.22. The contextual nature of cognitive processes in simple tasks.

FIG. 6.23. Two flight strips, each describing one aircraft. Column 1: (top) aircraft identification; (bottom) true airspeed/knots. Column 2: (top) previous fix. Column 3: (top) estimated time over next fix. Column 4: flight level (i.e., altitude in 100s of ft). Column 6: next fix.

FIG. 6.24. A sketch of the contextual cycle in relation to the knowledge base and the external environment. 
FIG. 6.25. Decrease in recall after a time interval, with different tasks during the retention interval (Posner \&. Rossman, 1965; copyright (C) 1965 by the American Psychological Association Reproduced with permission).

FIG. 6.26. Number of items recalled by air traffic controllers (data from Bisseret. personal communication, based on Bisseret, 1970).

FIG. 6.27 Recall of items about aircraft in different categories (based on data in Sperandio, 1970).

FIG. 6.28. Preflight planning by pilots with different levels of expertise (translated from Amalberti, 1992).

FIG. 6.29. Multitasking by a pilot during one phase of the flight (translated from Amalberti, 1992).

FIG. 6.30. Some of the links in a small part of a pilot's knowledge base (thick arrow indicates Cause-effect relation).

FIG. 6.31. Cognitive processing capacities change during the day. The different patterns of change suggest these capacities have different mechanisms. Reproduced from Folkhard, 1990, Circadian performance rhythms, in Broadbent et al (Eds.), Human Factors in Hazardous Situations (pp. 543-553), copyright (C) 1990 Clarendon Press, reproduced by permission of Oxford University Press. 
FIG. 6.32. "Inverted U" relation between internal arousal level and performance (it is not possible to account for the effect of all stressors in this way).

FIG. 6.33. Effect of changing working methods on relation between mental work and task work (Part A from Sperandio. 1972). This figure is a simplification: in practice the use of methods overlaps, so there are not discontinuities.

FIG. 6.34. Choice of optimum working method depends on task and personal factors. 